NISTIR 8089

\title{
An Industrial Control System Cybersecurity Performance Testbed
}

Richard Candell

Timothy Zimmerman

Keith Stouffer

This publication is available free of charge from: http://dx.doi.org/10.6028/NIST.IR.8089

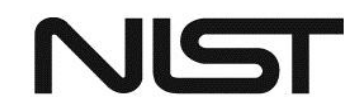

National Institute of Standards and Technology U.S. Department of Commerce 
NISTIR 8089

\title{
An Industrial Control System Cybersecurity Performance Testbed
}

\author{
Richard Candell \\ Timothy Zimmerman \\ Keith Stouffer \\ Intelligent System Division \\ Engineering Laboratory
}

This publication is available free of charge from:

http://dx.doi.org/10.6028/NIST.IR.8089

November 2015

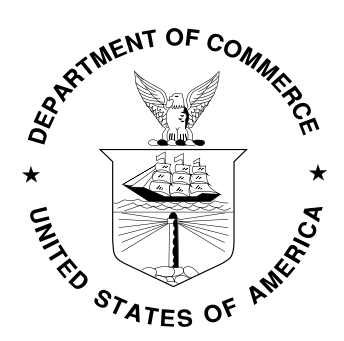

U.S. Department of Commerce

Penny Pritzker, Secretary

National Institute of Standards and Technology Willie May, Under Secretary of Commerce for Standards and Technology and Director 



\section{Table of Contents}

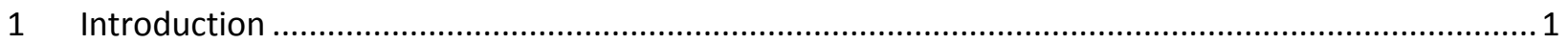

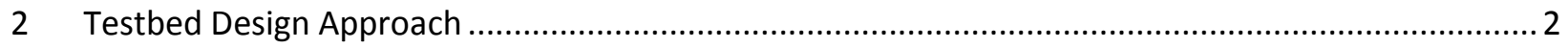

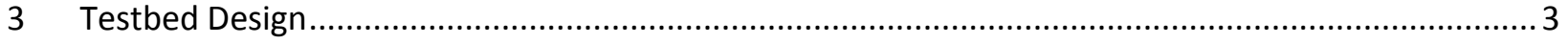

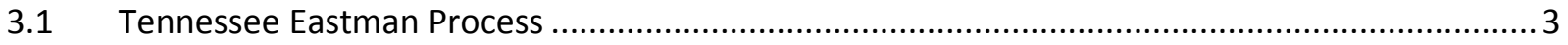

3.1.1 Tennessee Eastman Process: Analog Simulation ............................................................... 3

3.1.1.1 Tennessee Eastman Process: C++ Derivative .............................................................. 5

3.1.2 Testbed Enclave: Hardware-in-the-Loop Simulator......................................................... 5

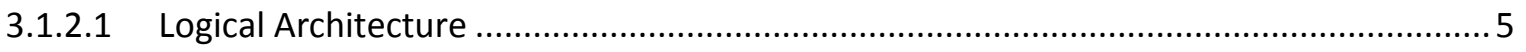

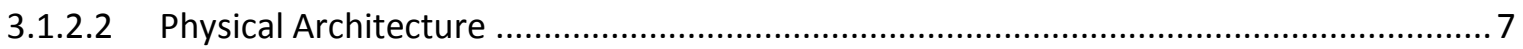

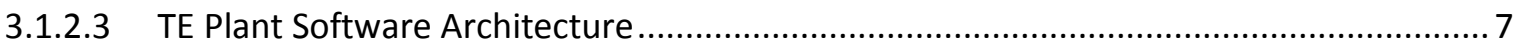

3.1.2.4 TE Controller Software Architecture ......................................................................... 8

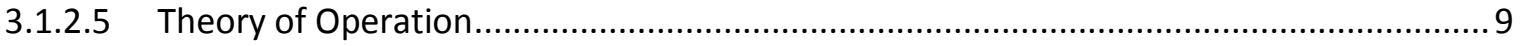

3.1.3 Process Control with Faster Dynamics..........................................................................

3.2 Cooperative Robotic Assembly for Smart Manufacturing ...................................................... 10

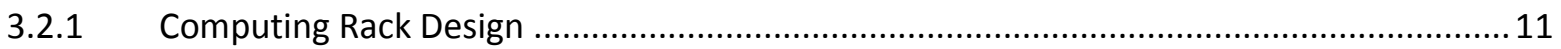

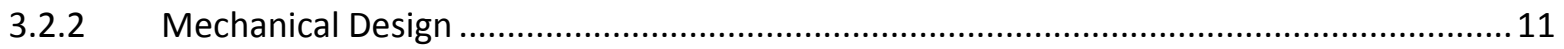

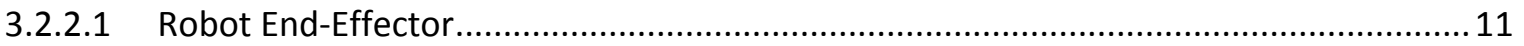

3.2.3 Receptacle for Machining Stations …............................................................................. 12

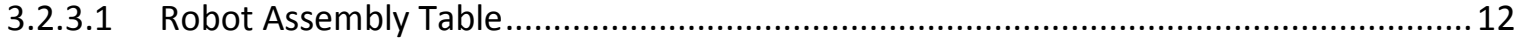

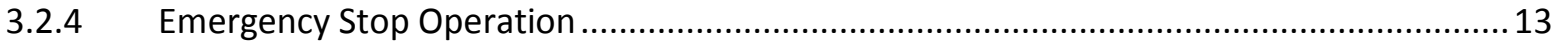

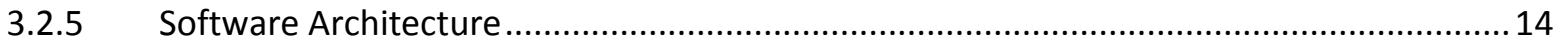

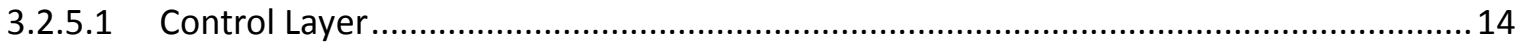

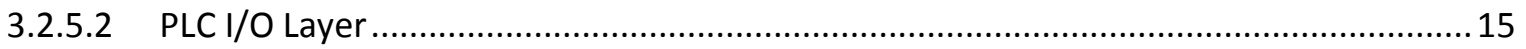

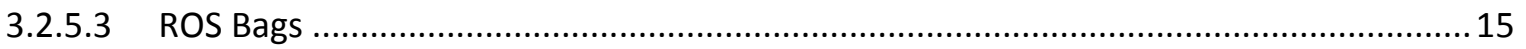

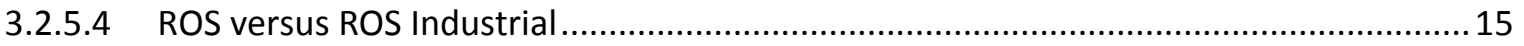

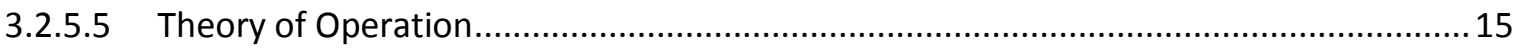

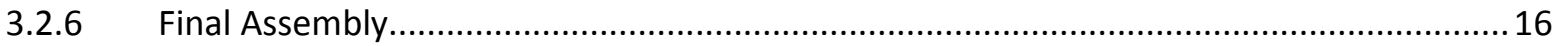

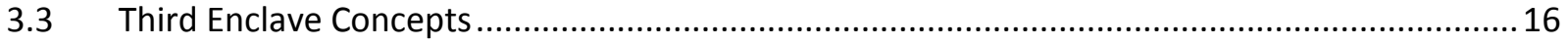

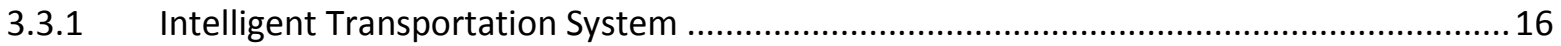

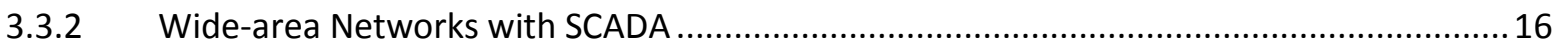




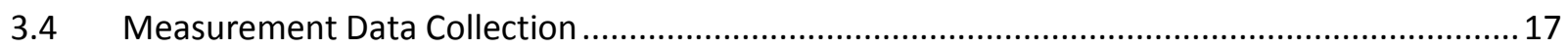

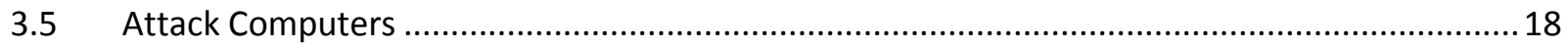

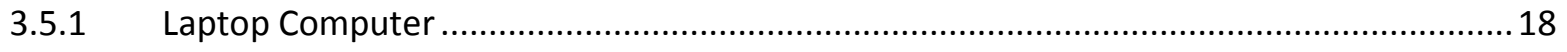

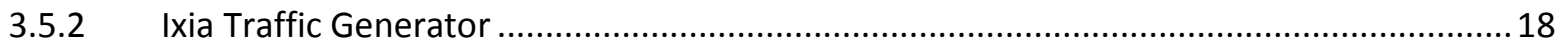

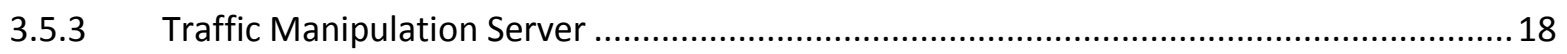

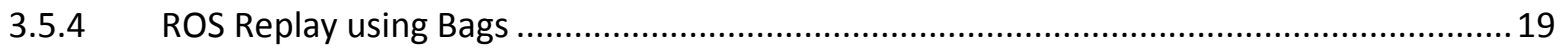

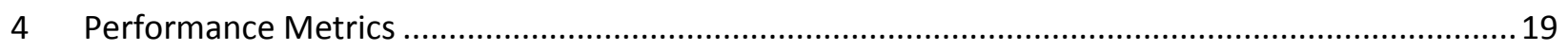

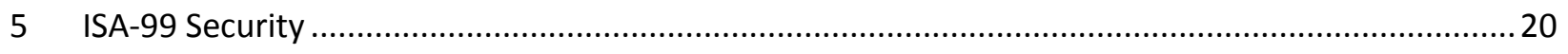

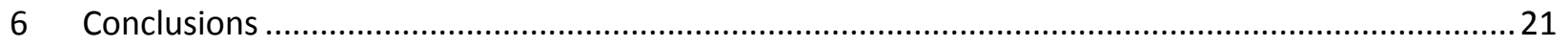

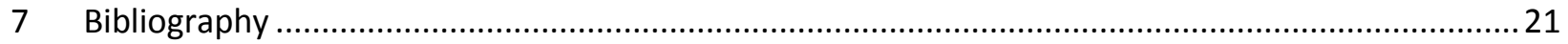

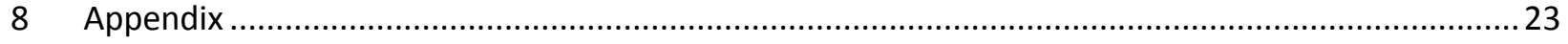

8.1 Glossary

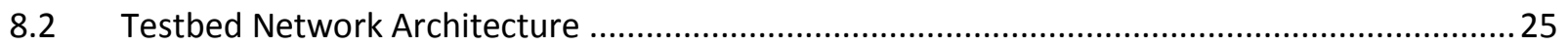

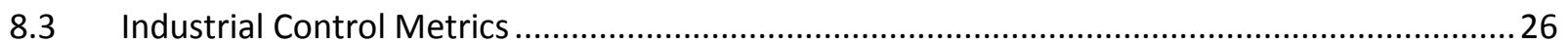

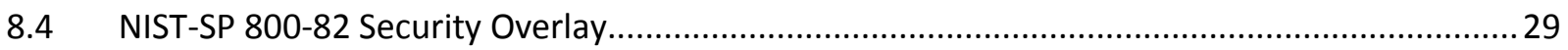

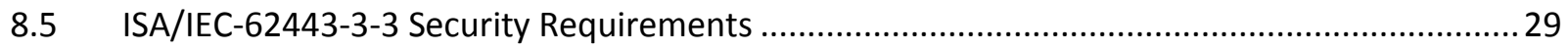

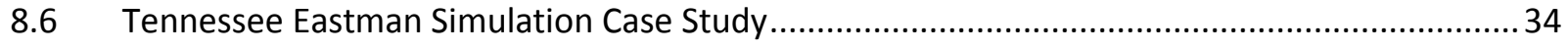

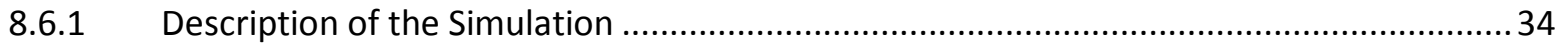

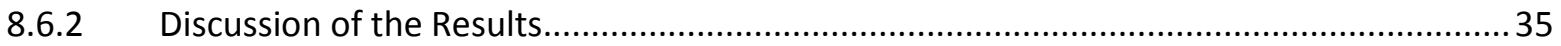

8.6.3 Conclusions and Recommendations .......................................................................... 47 


\section{List of Tables}

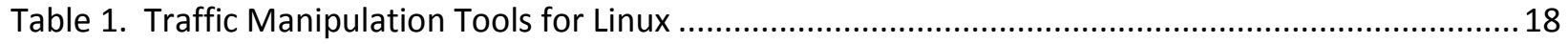

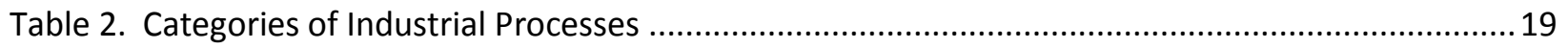

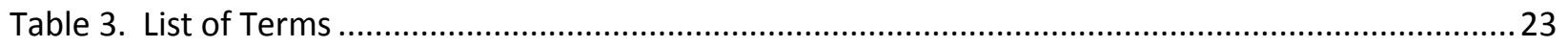

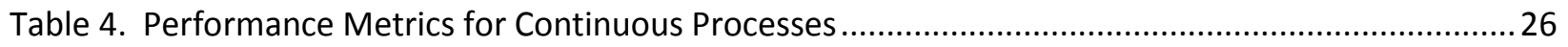

Table 5. Performance Metrics for Discrete Processes .........................................................................26

Table 6. Metrics for Measuring System Performance ...................................................................... 27

Table 7. Nominal System Properties for Measuring System Performance................................................2 27

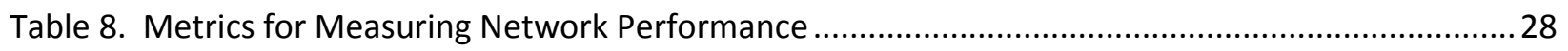

Table 9. ISA/IEC-62443-3-3 Requirements to Security Level Mapping ..................................................29

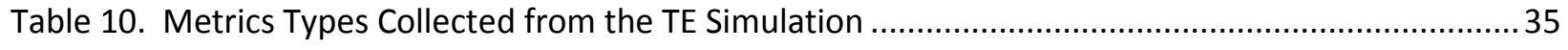

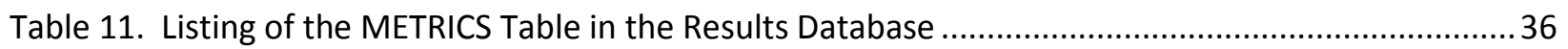




\section{Table of Figures}

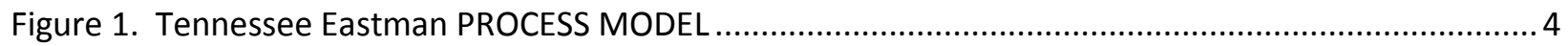

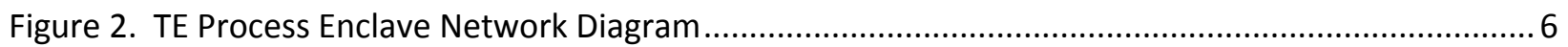

Figure 3. TE Simulator Physical Architecture Deployment ................................................................. 7

Figure 4. Software Architecture used for Communications between TE Plant and OPC.......................... 8

Figure 5. Software Architecture used for OPC Communications with the Controller .............................. 9

Figure 6. Robotic Assembly System Network Diagram ..................................................................... 10

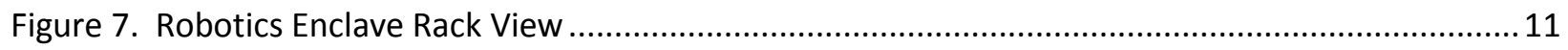

Figure 8. Spherical object end-effector for robotic enclave............................................................ 12

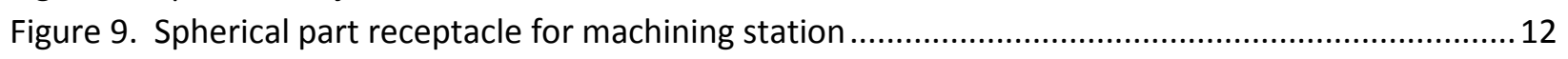

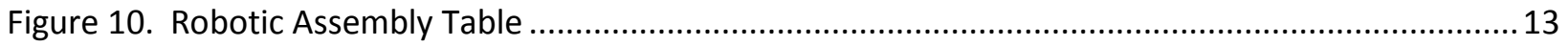

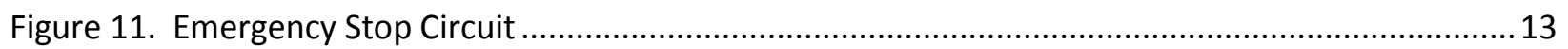

Figure 12. Robotics Enclave Node Level Software Architecture ...................................................... 14

Figure 13. Fully assembled discrete manufacturing robotics enclave.................................................16

Figure 14. Measurement Enclave Rack Deployment ............................................................................. 17

Figure 15. ISA/IEC-62443 Organization of Standards Documents ....................................................... 20

Figure 16. System Context for the Tennessee Eastman Enclave ........................................................ 25

Figure 17. Tennessee Eastman Simulation Architecture using a Gilbert-Elliot Channel Model ................34

Figure 18. Simple Gilbert Channel Model for a Generic Network Device................................................ 35

Figure 19. Reactor Pressure Metrics for All Disturbances ................................................................ 41

Figure 20. Standard Deviation of the Maximum Reactor Pressure Deviation for All Disturbances .......... 43

Figure 21. Product Quality Metrics for All Disturbances ................................................................... 44

Figure 22. Operating Cost Metric for All Disturbances .................................................................... 45

Figure 23. Deviation of Operating Cost from Baseline ...................................................................... 46 


\section{Introduction}

The National Institute of Standards and Technology (NIST) is developing a cybersecurity performance testbed for industrial control systems. ${ }^{1}$ The goal of the testbed is to measure the performance of industrial control systems (ICS) when instrumented with cybersecurity protections in accordance with the best practices and requirements prescribed by national and international standards and guidelines. Examples of such standards and guidelines include ISA/IEC-62443 and NIST Special Publication 800-82 [1].

The purpose of the testbed is to emulate real-world industrial systems as closely as possible without replicating an entire plant or assembly system. The testbed simulates a variety of industrial scenarios that include processes with slow dynamics and others with fast dynamics. Classification of faster versus slow is a relative determination and depends on the type of measurements being made. A slow process is one in which changes to state occur gradually over time. Processes with fast dynamics will exhibit a noticeable change of state soon after the system is perturbed.

Various industrial protocols are employed throughout the testbed including IP-routable and nonIP-routable protocols. Routable protocols include Internet Protocol (IP)-based protocols (e.g., Transmission Control Protocol (TCP) and User Datagram Protocol (UDP)) as well as industrial application layer protocols (e.g., EtherNet/IP, Open Platform Communication (OPC), and Modbus/TCP).

Non-IP-routable protocols include legacy fieldbus protocols, such as DeviceNet. The use of nonroutable protocols allows a deeper investigation of cybersecurity with fieldbus protocols and the controllers that make use of them; however, it was determined during the NIST Road mapping Workshop on Industrial Control Systems Cybersecurity that non-routable protocols were of lower priority than routable protocols. Non-routable protocols were designed to be open conduits for data flow; they were not designed for secure communications. It is unlikely that these types of legacy protocols will be modified to include security protections such as authentication and encryption. This design "limitation" makes these protocols good candidates for perimeter-based security mechanisms.

Each industrial scenario is a separate enclave within the testbed, as shown in Figure 16. The first of these scenarios is the Tennessee Eastman (TE) problem presented by Downs and Vogel [2], which is a well-known control systems problem in chemical process manufacturing. The TE problem is an ideal candidate for cybersecurity investigation because it is an open-loop unstable process and requires closedloop control to maintain process stability and optimize operating costs. The TE process can be considered a process with slow dynamics in relation to the information update rate of the control network. These slow dynamics enable an adversarial agent to compromise the control infrastructure and remain undetected for a significant duration. Attacks that actively evade detection (stealth attacks) or attacks that exploit specific dynamic properties of the system (geometric attacks) [3] are particularly effective against the TE process.

\footnotetext{
${ }^{1}$ Disclaimer: Certain commercial equipment, instruments, or materials are identified in this paper in order to specify the experimental procedure adequately. Such identification is not intended to imply recommendation or endorsement by the National Institute of Standards and Technology, nor is it intended to imply that the materials or equipment identified are necessarily the best available for the purpose.
} 
The second scenario is a robotic assembly system in which industrial robots work together cooperatively to accomplish the task of moving parts through a simulated manufacturing operation. The robots work cooperatively according to a plan that changes dynamically based on process feedback. The robotic enclave includes two small industrial grade robots and a supervisory Programmable Logic Controller (PLC) with safety processing.

The third enclave will be designed by Vanderbilt University under a cooperative research agreement with NIST. Several concepts were proposed for the third enclave including a pipeline network with a wide area network Supervisory Control and Data Acquisition (SCADA) infrastructure and an intelligent transportation system with distributed infrastructure control. A wide area network would complement the local area network scenarios covered by the other two enclaves.

\section{Testbed Design Approach}

Industrial control systems (ICS) can be classified as networks that include process control systems, robotic manufacturing systems, home and office building automation systems, intelligent transportation systems, advanced aircraft, and spacecraft [1]. These types of networks are often composed of numerous interconnected devices with centralized or decentralized control depending on the application. Modern requirements of modularity, decentralization, ease of maintenance, and lower operational costs have driven designers of network control systems toward the adoption of routable data communications protocols traditionally found in home and office environments, such as Ethernet (IEEE 802.3) and Wi-Fi (802.11). With this change, industrial control systems security has become increasingly important. Traditional information technology (IT) security policies focus primarily on confidentiality with network availability being least important. In contrast, ICSs that are part of critical infrastructure must maintain a high level of system availability and operational resilience for many reasons including economic, environmental, human safety, and national security. For many processes, it would be unacceptable to degrade performance for the sake of security. A risk/benefit analysis is required for each system to make such a determination. Security protections must be implemented in a way that maintains system integrity during normal operation as well as during times of cyber-attack. Indeed, ICS security must include elements of resilient physical design (e.g., redundancy and physical adaptability) in addition to network security to maintain required system availability. Such requirements are determined by a process of careful risk analysis and system engineering. The ICS testbed serves as a test platform to provide guidance on how to implement security in an ICS, based on sound measurement science.

The ICS cybersecurity testbed is designed to demonstrate the application of security to a variety of processes, such as control of a chemical plant, dynamic assembly using robots, and distributed supervision and control of large wide-area networks (e.g., gas pipelines, water distribution pipelines, and distributed intelligent transportation systems). As stated, the primary objective of the testbed is to demonstrate the application of industrial control system security standards, such as NIST SP 800-82 [1] to a networked control system and measure the performance degradation or improvement, if any, after applying the protections. The testbed will also serve as a guide on how to implement security safeguards effectively without negatively affecting process performance. In addition, while no system can be made completely secure from network attacks [3], a secondary objective of the testbed is to measure the performance of industrial control systems while undergoing cyber-attack. Resiliency will be a central focus of systems under attacks. The testbed will be made available to academia, government, and 
industry to conduct analysis of new technologies that are designed to improve intrusion detection technologies and make processes more resilient to attacks. The testbed will support research for a period of five years. Penetration testing will be conducted during the latter years of the ICS security research project.

Various commercial products exist to protect systems that use industrial protocols. Products such as the Tofino Security Appliance and the CISCO Adaptive Security Appliance (ASA) firewall devices provide feature-rich security capabilities (e.g., content security, encryption, identity authentication, user authorization, and intrusion prevention). These products are designed primarily to protect PLCs from network perimeter exploitations. While these technologies do supply valuable network protection, they do not provide (as per their designs) the ability to measure network performance as a function of packet flight metrics. These metrics include average packet flight time (delay), packet flight time uncertainty (jitter), and payload integrity (noise). Therefore, the initial measurement of each enclave will be based on artificially induced delay, jitter, and noise; performance of the processes being studied will be examined as a function of those parameters.

\section{Testbed Design}

\subsection{Tennessee Eastman Process}

\subsubsection{Tennessee Eastman Process: Analog Simulation}

The Tennessee Eastman (TE) control problem was chosen as the continuous process model for a number of reasons. First, the TE model is a well-known plant model used in control systems research and the dynamics of the plant process are well-understood. Second, the process must be controlled otherwise perturbations will drive the system into an unstable state. The inherent unstable open-loop operation of the TE process model presents a real-world scenario in which a cyber-attack could represent a real risk to human safety, environmental safety, and economic viability. Third, the process is complex, nonlinear, and has many degrees of freedom by which to control and perturb the dynamics of the process. And finally, numerous simulations of the TE process have been developed with readily available reusable code. We chose the University of Washington Simulink controller design by Ricker [2]. The Ricker Simulink model was chosen for its multi-loop control architecture, making distributed control architectures viable. It accurately matches the Downs and Vogel model, and the control code is easily separable from the plant code.

The TE process model is illustrated in Figure 1. Downs and Vogel did not reveal the actual substances used in the process, but instead they used generic identifiers for each. The process produces two products, $\mathrm{G}$ and $\mathrm{H}$, from four reactants $\mathrm{A}, \mathrm{C}, \mathrm{D}$, and $\mathrm{E}$. The process is defined as irreversible and exothermic, and the reaction rates of the four reactants are a function of the reactor temperature. The process is broken into five major operations, which include a reactor, a product condenser, a vapor-liquid separator, a product stripper, and a recycle compressor.

The process is described in detail in [2], however, a synopsis is given as follows. Gaseous reactants are combined in the reactor to form liquid products. The reactor temperature must be controlled and is cooled using cold water cooling bundles. The reaction is not $100 \%$ efficient and some gaseous feed components remain. The output of the reactor is fed to a condenser where the products are further cooled into liquid form. The vapor-liquid separator then separates unreacted gases from the liquid 
products. The unreacted gases are sent back to the reactor by a centrifugal recycle compressor. Again, the separation process is not $100 \%$ efficient, and the remaining reactants are removed in a stripping column by stripping the mixture with $C$ in feed stream four (4). The products, $G$ and $H$, are then sent downstream for further refining. Byproducts of the process are purged from the process through the purge valve of stream nine (9).

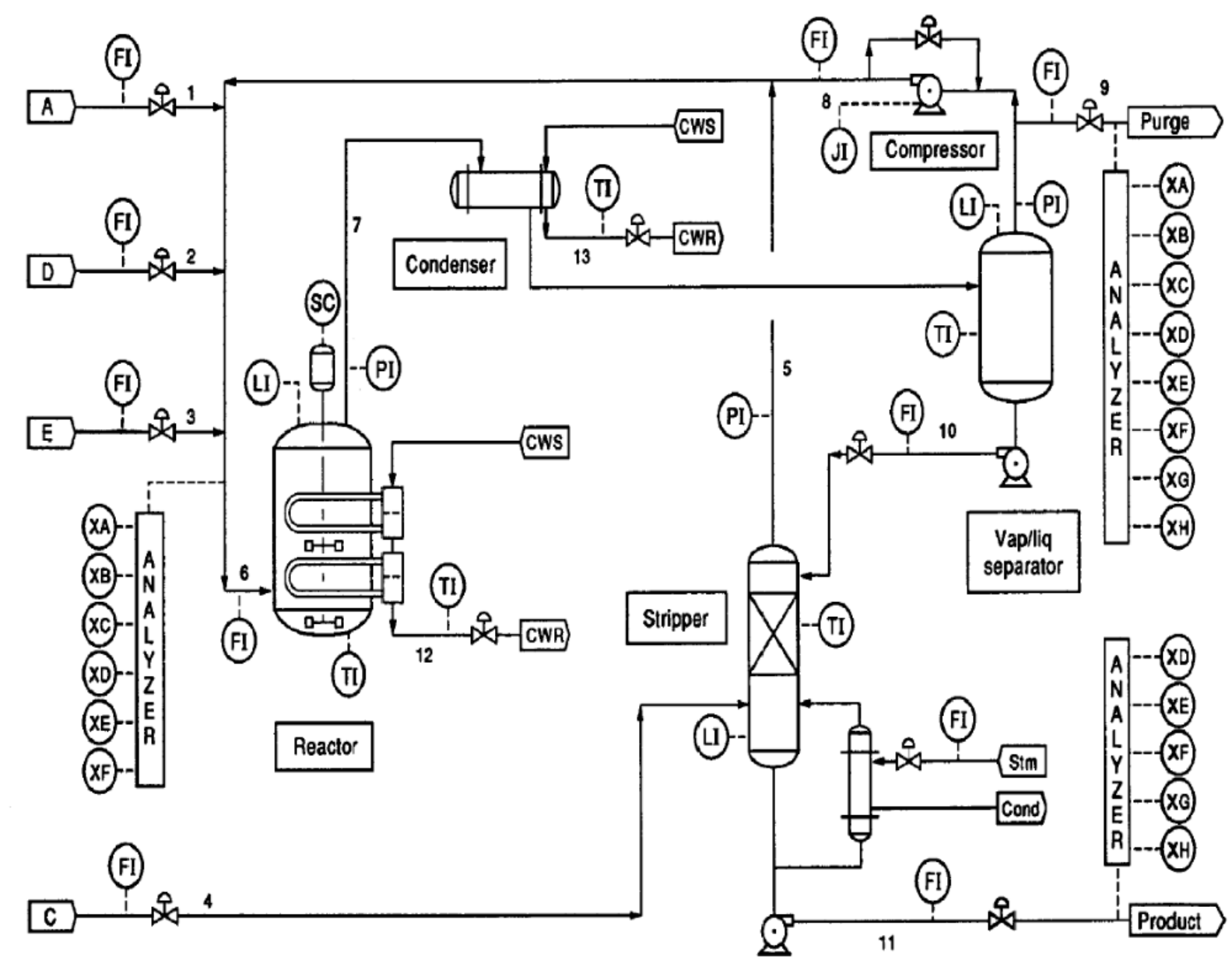

Figure 1. Tennessee Eastman PROCESS MODEL

The process has six (6) different modes of operation which control the $\mathrm{G} / \mathrm{H}$ mass ratio and the production rate through stream eleven (11). Our primary use case for the system will be the base case, indicated as Mode 1. Downs and Vogel provide heat and material balance data for the Mode 1 case. It is important to note that the process is designed to shut down if the reactor pressure exceeds $3000 \mathrm{kPa}$; however, as noted in [3], the reaction efficiency improves as the reactor pressure increases. This indicates that the reactor pressure will be driven as close to the maximum threshold without exceeding the shutoff limit. The reactor pressure set-point presents a security vulnerability. The reactor pressure will be driven as close as possible to the maximum limit with little margin for error. It is conceivable that an attacker could target the reactor pressure using a geometric attack or a surge attack combined with a human 
machine interface (HMI) spoofing attack. Krotofil and Cardenas [3] provide an excellent discussion of the TE process and potential security vulnerabilities.

The plant and controller have been separated such that the two processes may be executed on separate machines with communications conducted via an arbitrary network protocol, such as TCP, UDP, OPC, or EtherNet/IP. For an analog analysis of performance, a network connection is unnecessary, and instead a basic delay system may be inserted to simulate the effects of the communication channel. The communication channel is shown in the Simulink diagram between the TE Controller and the TE Plant. The communications channel simulates the packet error rate and delay variation of the channel itself. By introducing the channel, it will be possible to predict in simulation the effect of the channel on the performance of the system. This analysis will be used to predict the performance of the TE chemical process when cybersecurity protections are applied.

\subsubsection{Tennessee Eastman Process: C++ Derivative}

In order to facilitate the transfer of the TE plant and controller models across different enclaves, the original Fortran code of the plant model from Downs and Vogel and the Simulink controller by Ricker were converted to $\mathrm{C}$. Like the Simulink model, the plant and controller processes are separated, allowing the execution of the two processes on separate hardware and simulation of the communications channel.

The $\mathrm{C}++$ models are wrapped in a feature-rich, command-line application that allows modification of run-time options and parameters, as well as enclave specific options. For example, for enclaves requiring real-time operation of the model, the '- $r$ ' switch is included on the command line at execution. The high-level application layer and modular architecture of the $\mathrm{C}++$ code allow for seamless integration and software updates to all enclaves running the models.

The code is available on GitHub: https://github.com/usnistgov/tesim

\subsubsection{Testbed Enclave: Hardware-in-the-Loop Simulator}

While a mathematical simulation is an important first step in the analysis of the performance of any system, it is equally important to understand how a practical system behaves when instrumented with security protections that will invariably insert packet flight uncertainty. A hardware-in-the-loop (HIL) simulator was constructed to demonstrate the impacts of cybersecurity on the performance of a manufacturing process [4].

\subsubsection{Logical Architecture}

The simulator is intended to be reconfigurable such that various network topologies, cybersecurity hardware, and simulation models may be hosted and evaluated. For the TE process, the enclave is partitioned in accordance with the baseline case shown in Figure 2. 


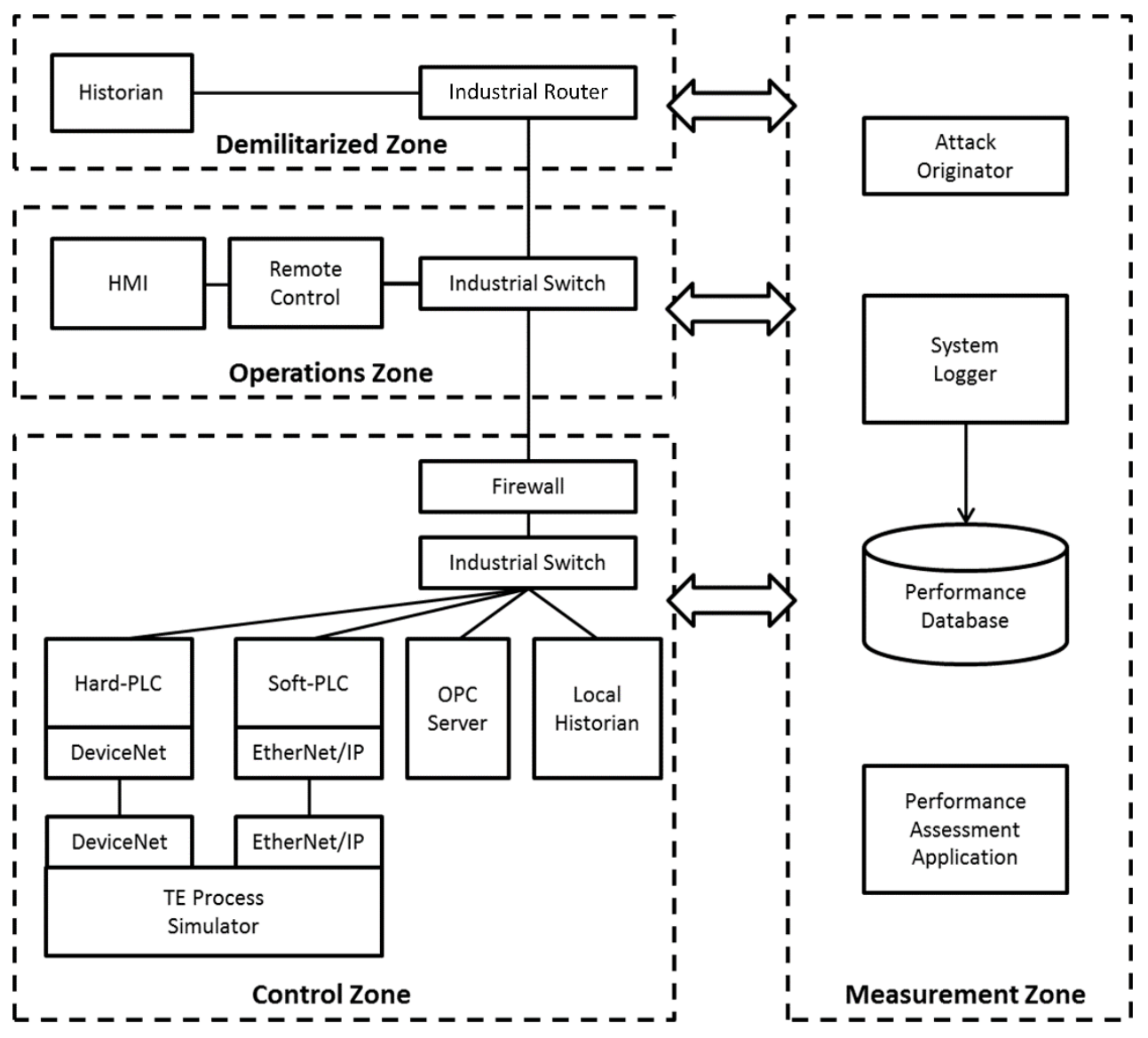

Figure 2. Te Process Enclave Network Diagram

The system is separated into three zones: a control zone, an operations zone, and demilitarized zone (DMZ). The control zone hosts the TE plant and controller processes. The operations zone hosts the human machine interface for visualizing the process data, and manipulating the simulation set-points and parameters. The DMZ separates the control network from the business network, and contains a historian to allow business-level access to plant and controller state data without allowing access to the control network.

State data between the plant and controller processes is handled through the OPC server using one or more industrial protocols via the PLC, such as the non-routable protocol DeviceNet, or the routable protocol EtherNet/IP. Process states are transmitted from the PLC to the OPC server for distribution, while the local historian records the state data and replicates it to the enterprise historian in the DMZ.

The firewall separating the operations zone from the control zone implements deep packet inspection and device authentication (white listing), which will be used as a PLC cyber-protection mechanism.

The TE enclave also contains network patches to the measurement enclave to facilitate packet capture and the implementation of custom network delay mechanisms that serve to emulate the delays introduced by security devices. 


\subsubsection{Physical Architecture}

Shown in Figure 3 is an illustration of the TE Process Simulator physical architecture. The TE Enclave is a full height 19" rack with various computing, storage, and control components. Computing resources support the plant simulator, controller simulator, OPC server, historian, and HMI. A video management system is included to support process visualization and user input across all computing resources.

Power in the TE rack is supplied by an uninterrupted power supply (UPS) capable of supplying power at $100 \%$ load for at least 5 minutes. This will protect the enclave from damage or interruption due to intermittent power failures. It is expected that power outages of any duration are unlikely at the NIST Gaithersburg campus. The enclave includes an industrial switching center, a hard-PLC center, and a softPLC (PC-based) center. The switching center includes an Allen-Bradley Stratix 8300 industrial router and two Allen-Bradley Stratix 5700 industrial switches.
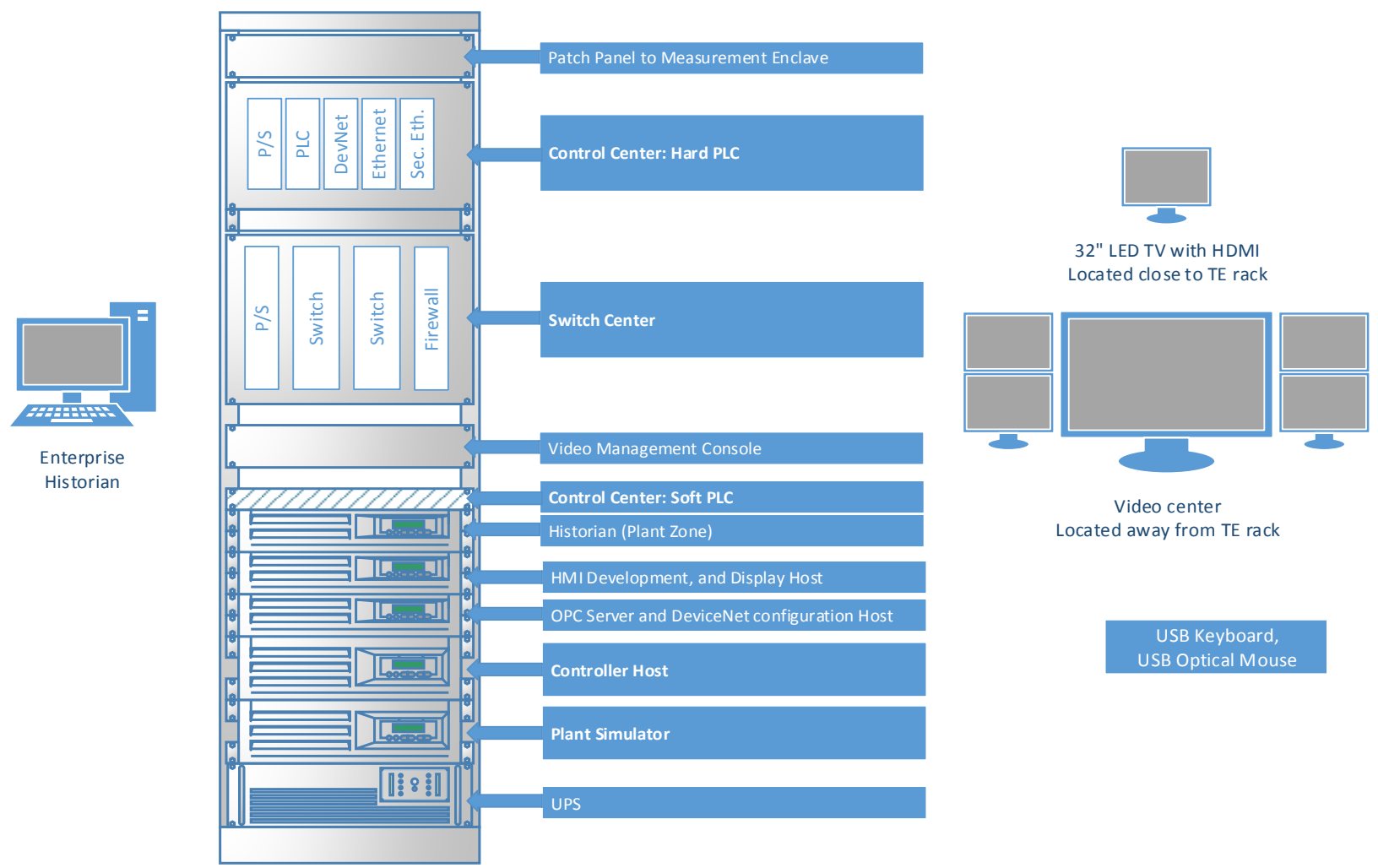

Figure 3. Te Simulator Physical Architecture Deployment

\subsubsection{TE Plant Software Architecture}

An illustration of the software architecture for the plant is shown in Figure 4. Communications between the plant simulation and the industrial network is conducted through a simple architecture that can be adapted for any industrial protocol. Two card-based MOLEX hardware products are used to 
perform the DeviceNet and EtherNet/IP network emulation: a MOLEX SST-DN4MS-PCU DeviceNet Multidrop Slave (DNMSLAVE) and a MOLEX DRL-EIP-PCIE EtherNet/IP.

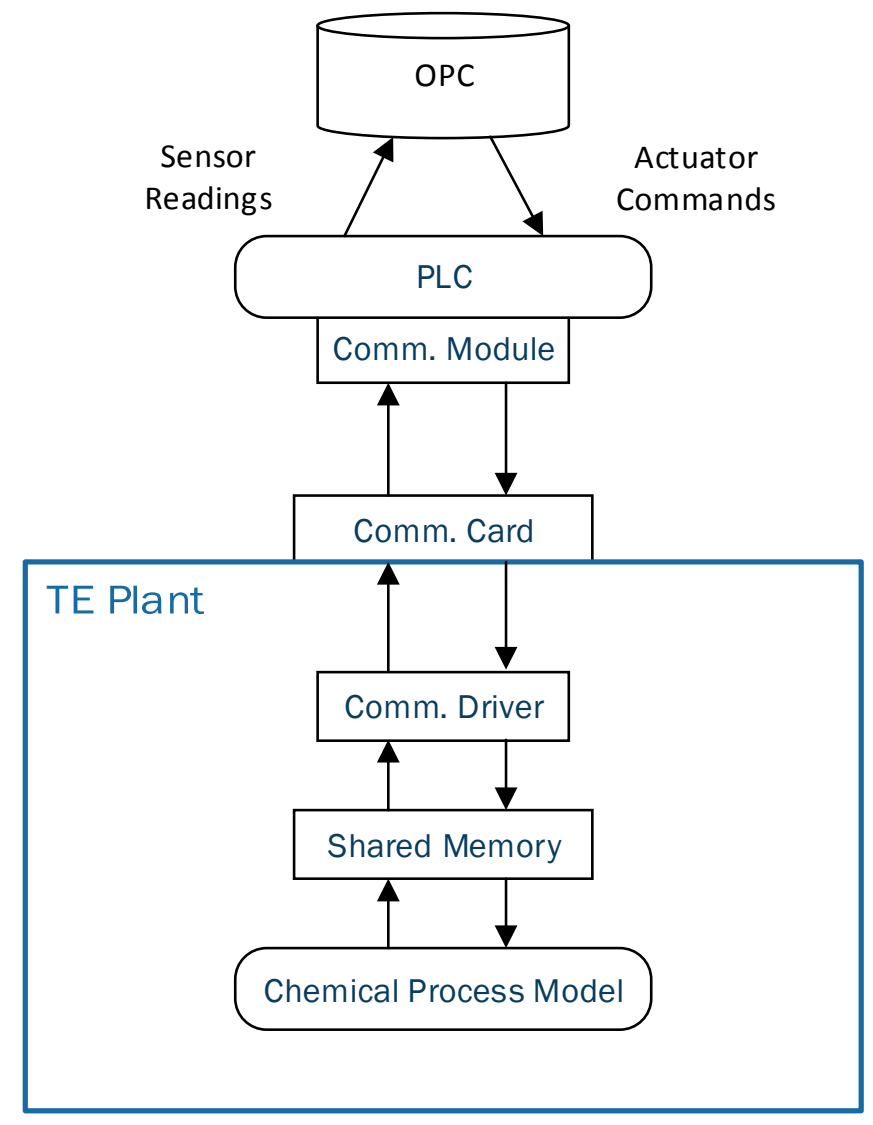

Figure 4. Software Architecture used for Communications between te Plant ANd OPC

The interface between the communications driver and the chemical process model is implemented using shared memory. The PLC obtains the process states from the communication card; each state is emulated as an individual device on the communication bus/network. For DeviceNet each state has a media access control identifier, and for Ethernet/IP each state has a device identifier.

The PLC continuously polls the process states (sensor readings) from the communication bus/network and updates the corresponding OPC tags for use by the controller process. The actuator commands resulting from the controller process are received by the PLC via scanning the OPC tags, and the states propagated back down through the architecture to emulated actuator devices on the communication bus/network for use on the next iteration of the chemical process model.

\subsubsection{TE Controller Software Architecture}

An illustration of the software architecture for the controller is shown in Figure 5. Communications between the Simulink controller and the industrial network are conducted via OPC.

OPC communications are conducted using Simulink's OPC Toolbox blocks, simplifying the implementation. Connections to the OPC Server are configured using the OPC Configuration block. The OPC Configuration block defines the OPC clients to be used in the model, configures pseudo real-time 
behavior for the model, and defines behavior for OPC errors and events. All error handling and queuing are handled by the toolbox.

On every control iteration, the controller pulls the current plant simulation states (sensor readings) from the OPC server, processes these states through the controller model, and returns the new controller states (actuator commands) to the OPC server for use by the plant model.

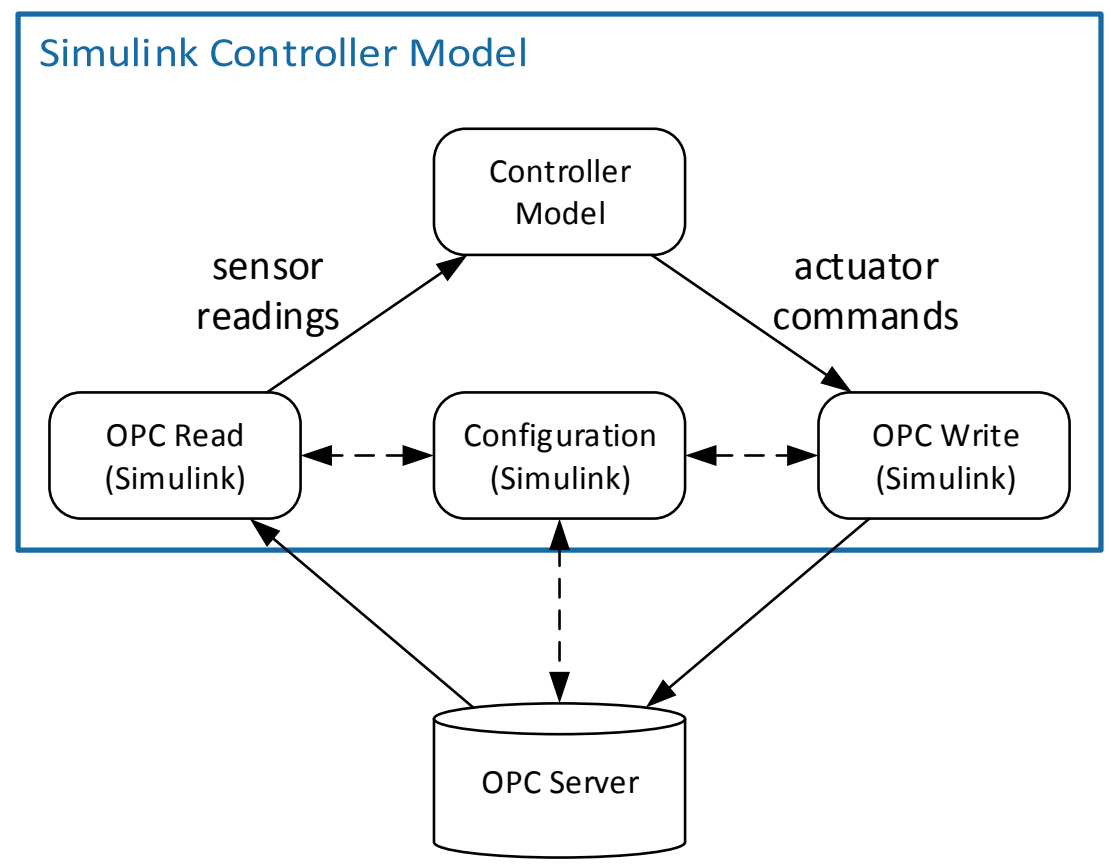

Figure 5. Software Architecture USEd for OpC Communications With the Controller

\subsubsection{Theory of Operation}

To begin the simulation, model parameters for the current simulation are loaded into state tags within the OPC server via the HMI. Since the plant and controller are located on multiple machines, a start/stop tag in the OPC server is used to initiate the simulation on both machines. Both the plant and controller will monitor this tag and will begin their simulations once it is set. This tag is set by an operator via the HMI.

Both the chemical process model and the controller receive the model states from the OPC server for each integration time step. At the time of writing this paper, the integration time step is set to 1.8 seconds. Each simulation runs asynchronously until the configured simulation time is reached, at which point both simulations will terminate.

\subsubsection{Process Control with Faster Dynamics}

While the Tennessee Eastman process is well understood and a good model to use for the implementation of security policies and technologies, the process itself is slow. The model operates with such slow time constants that changes in the process dynamics caused by induced model disturbances can take hours to display a noticeable effect.

The primary objective of the TE enclave is to validate security standards, measure their performance impacts, and provide feedback to the standards bodies that support them; and the TE 
process is well suited for this purpose. However, a secondary objective of the enclave is to create a system with a broad attack surface through which security penetration testing may be conducted and the effects researched. The slow dynamics of the TE process may present significant project management challenges, and a process with faster dynamic responses (on the order of seconds) would be required to facilitate more timely research.

\subsection{Cooperative Robotic Assembly for Smart Manufacturing}

The robotic assembly enclave demonstrates security in a discrete state process with fast dynamics and high data throughput demands using a combination of a deterministic real-time protocol and Ethernet-based IP protocols. The network design of the robotics enclave is shown in Figure 6 . The robotics enclave is designed as a local area network, using the EtherCAT real-time industrial protocol for communication between the controller and the robots.

The robotics enclave is designed similar to the TE enclave, such that different functions of the robotics system are encapsulated in more than one subnet. A layer 3 switch is used to facilitate rapid network configurability. As with the TE enclave, the robotics enclave serves to validate the requirements specified in the prevalent security standards.

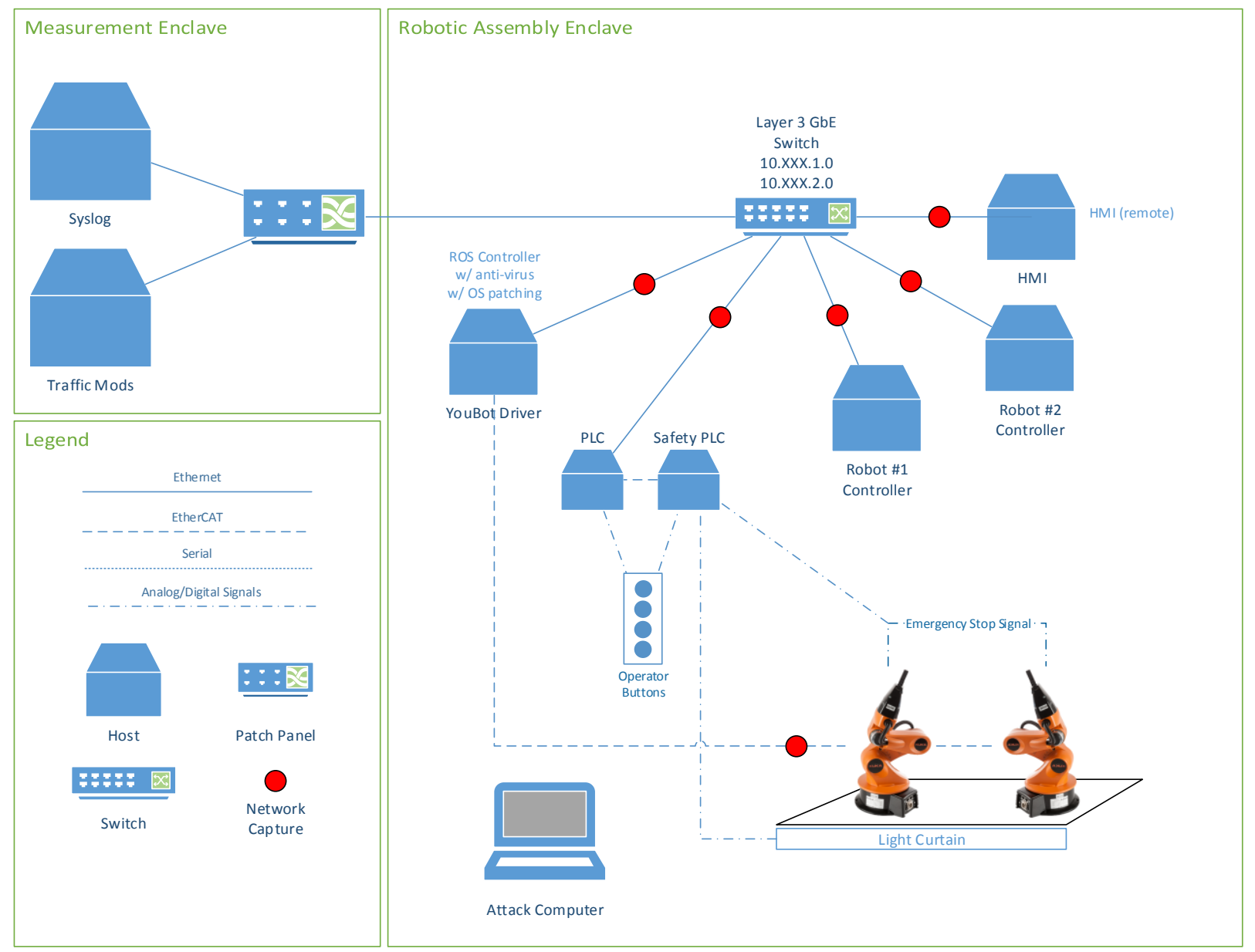

Figure 6. Robotic Assembly System Network Diagram 


\subsubsection{Computing Rack Design}

An illustration of the robotics rack is shown in Figure 7. A 42-U full-size 19" rack with UPS is used to house the computing resources. The rack includes a 24-port Ethernet patch panel and Layer 3 industrial switch. Security capabilities of the switch include integrated router/firewall/virtual private networking (VPN); stateful firewall with network address translation (NAT) support; IPSec VPN; and VPN with 3DES, AES128, AES256 support. Computing resources include rack-mounted servers for the two Robot Operating System (ROS) controllers (one for each robot). A tower computer is used for the ROS Core, robot driver, and remote shells.
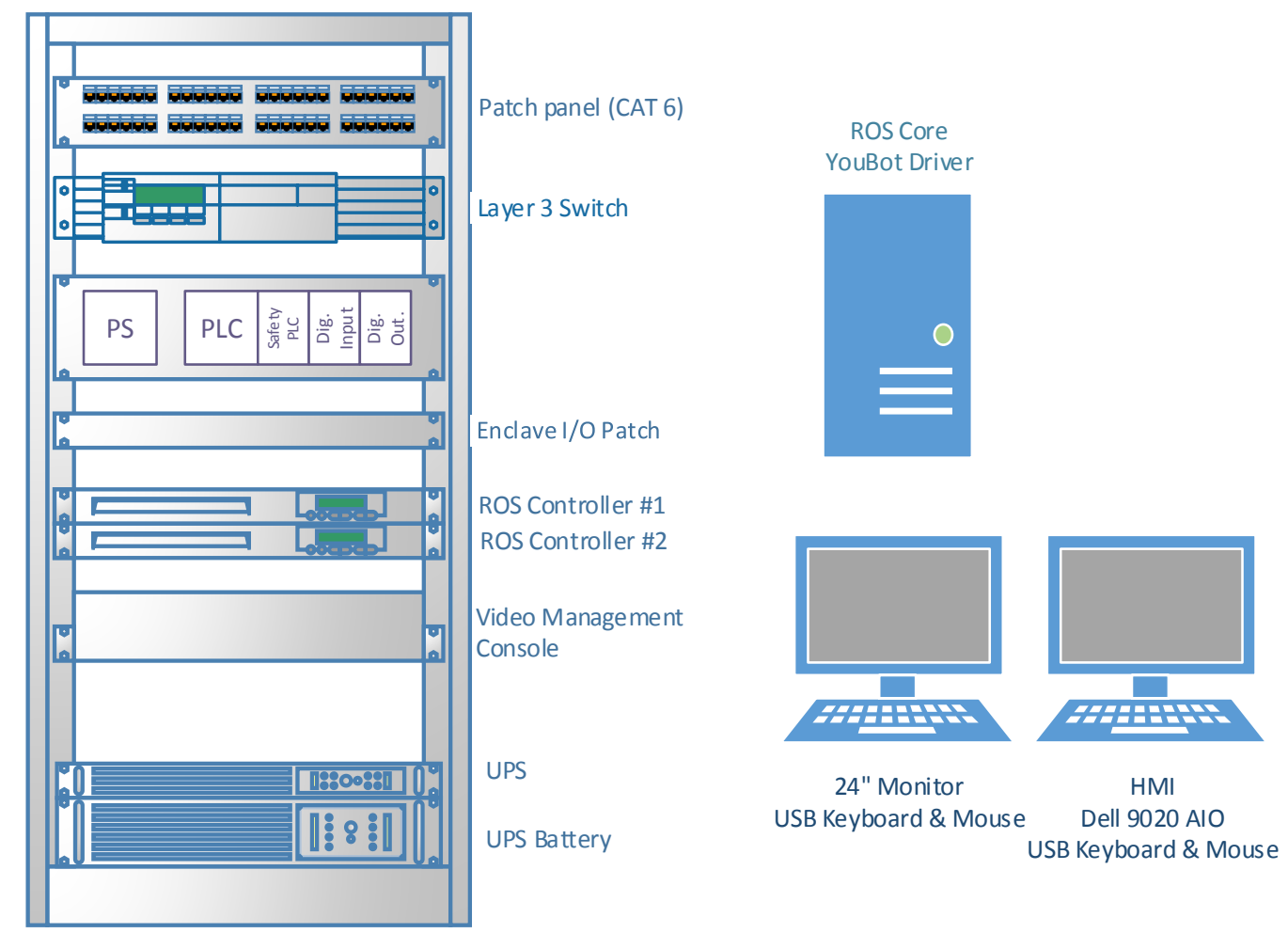

Figure 7. Robotics Enclave Rack View

The rack includes a control center that contains a PLC for enclave operations, a Safety PLC for monitoring safety inputs/outputs (I/O), and I/O modules for normal enclave signals (e.g., sensors and operator buttons).

\subsubsection{Mechanical Design}

\subsubsection{Robot End-Effector}

The robot end-effector is designed to pick-up spherical parts (e.g., golf balls) using a profile that is specific to the shape and size of the part, and 3-D printed. A diagram of the end-effector is shown in Figure 8. The shape allows for position repeatability of the part within the jaws relative to the endeffector, and is also capable of lifting the part from the machining station while performing the closing operation. 

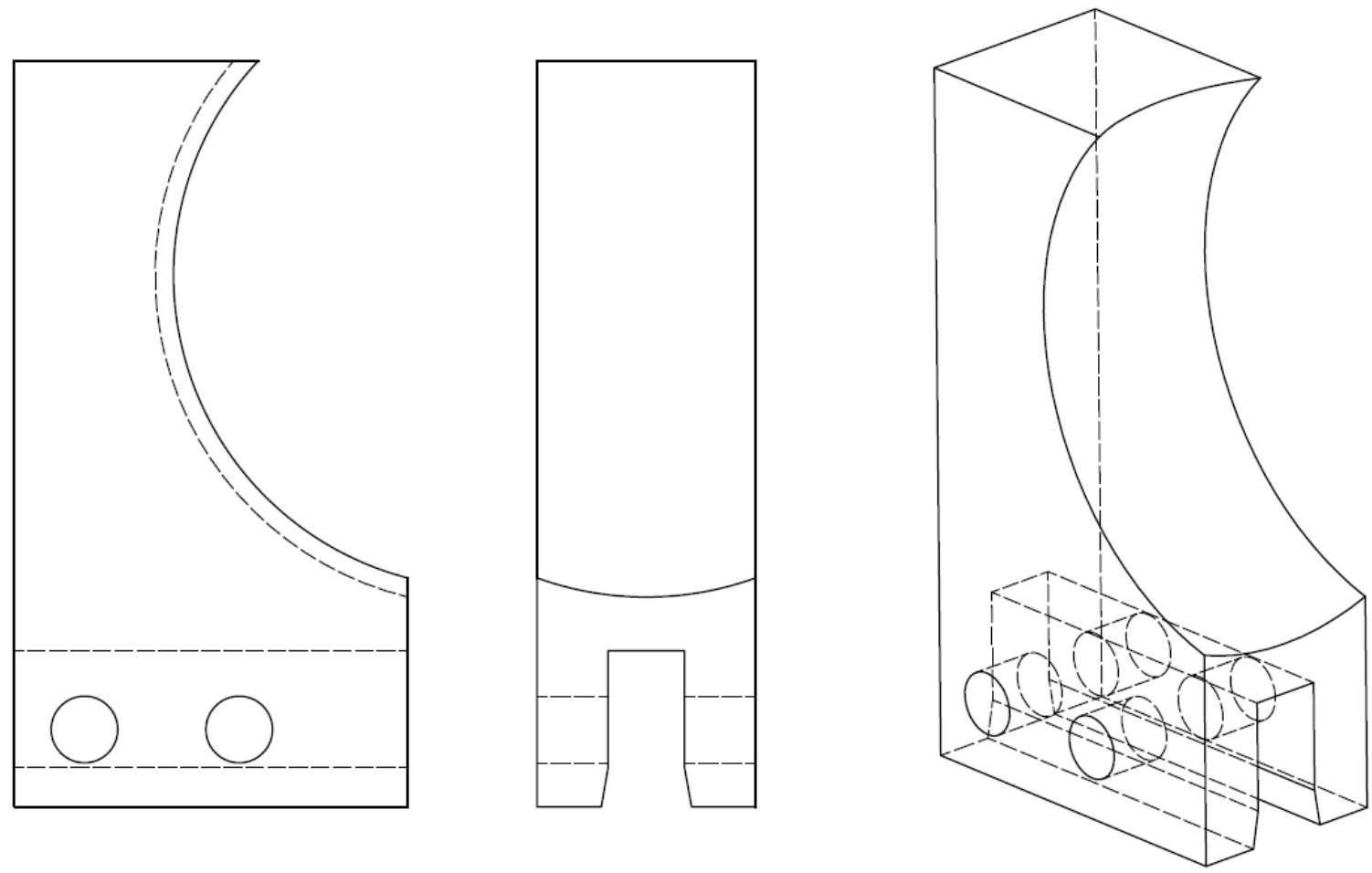

FigURE 8. SPHERICAL OBJECT END-EFFECTOR FOR ROBOTIC ENCLAVE.

\subsubsection{Receptacle for Machining Stations}

A special receptacle is used to receive and store spherical parts, as shown in Figure 9. Each receptacle was designed with an integrated infrared photoelectric sensor and is used to detect when a part is present in the receptacle. This information is sent directly to the PLC for distribution.

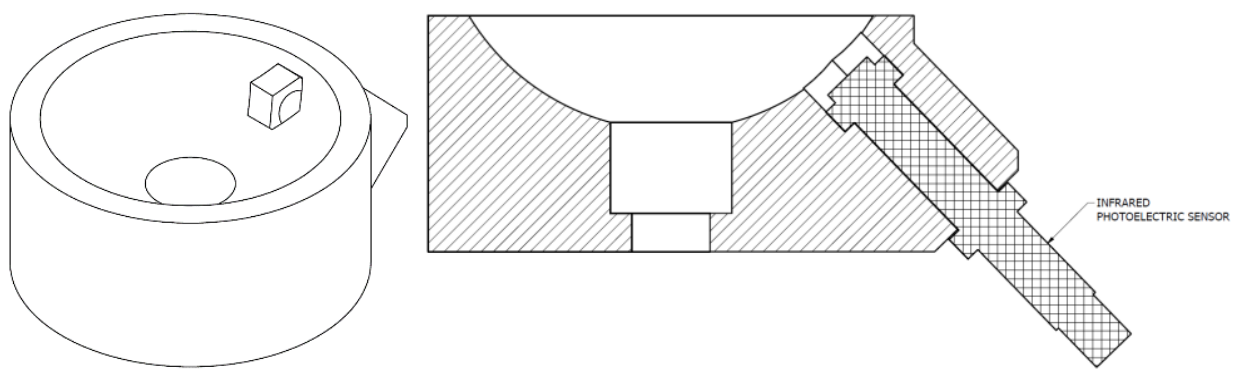

FIGURE 9. SPHERICAL PART RECEPTACLE FOR MACHINING STATION

\subsubsection{Robot Assembly Table}

An optical table serves as the assembly table upon which the two Kuka YouBot robots are stationed. A drawing of the station is shown in Figure 10. The robots are positioned at the center of the optical table, allowing them to interact with the machining stations and each other. The table is divided 
into three regions: a material queue, an operating zone for each robot to move parts through their associative machining stations, and a center cooperative zone for passing parts from one robot to the other. The part queue is located at the front of the optical table and gravity-feeds parts from one side of the table to the other. Parts in the queue are detected by an infrared photoelectric sensor integrated into the hard stop at the base.

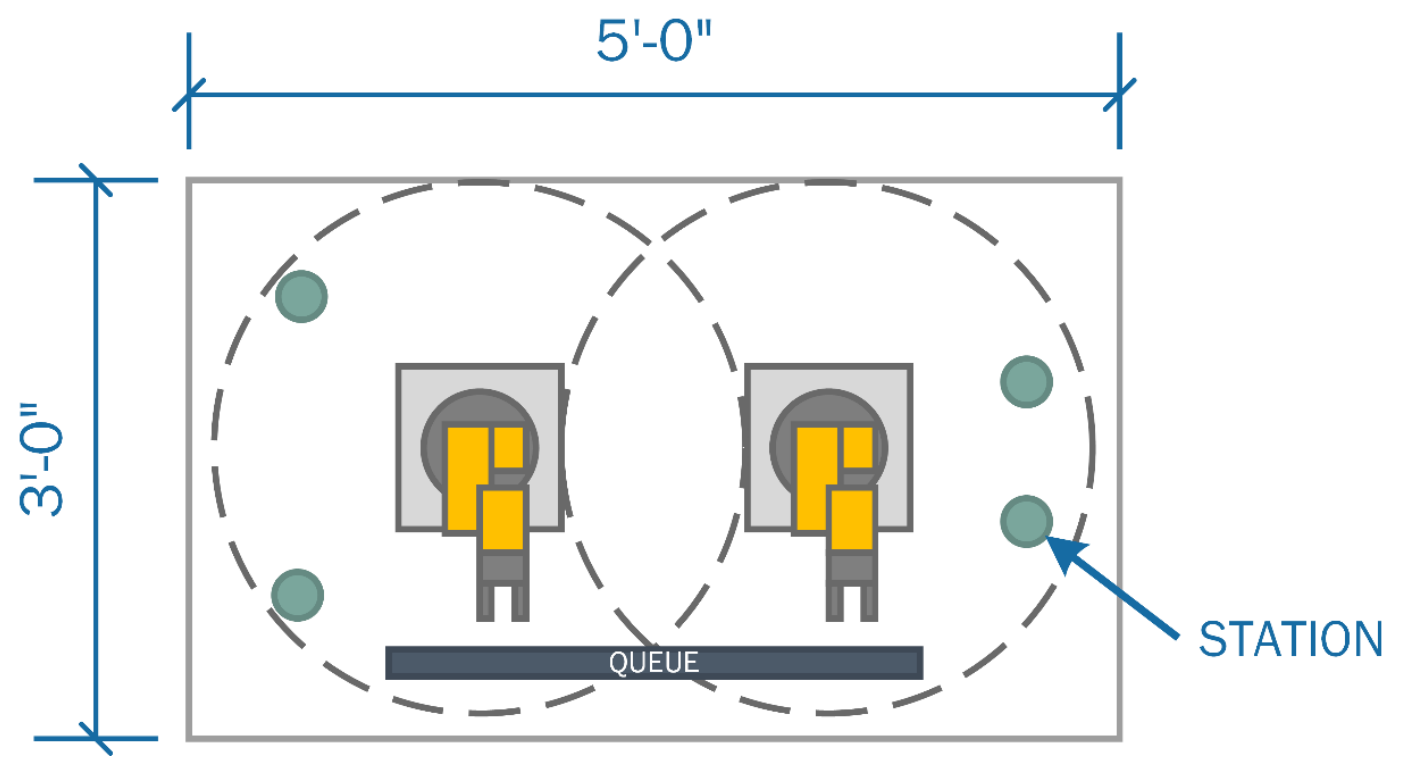

Figure 10. ROBOtic ASSEMbLY TABLE

\subsubsection{Emergency Stop Operation}

A safety PLC is used to monitor the status of the emergency stop button and light curtain. The light curtain serves to detect human entry at the front of the optical table. A safety relay handles the emergency stop signal for the robots. If an unsafe condition is detected by the safety PLC, the safety relay will de-energize, causing the robots to halt their operations using the robots's integrated emergency stop feature. When an operator puts the PLC into the "stop" mode, the emergency stop button and light curtain are bypassed to allow for human interaction with the stations and robots. A simplified ladder logic diagram of the safety program is shown in Figure 11.

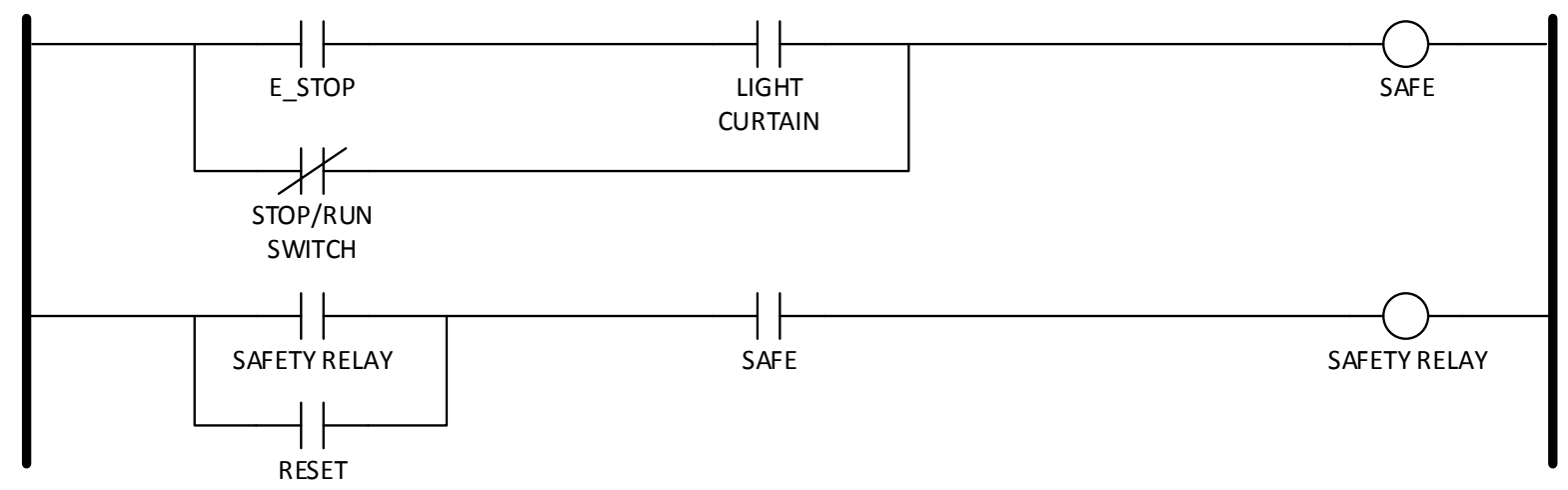

Figure 11. Emergency Stop Circuit 


\subsubsection{Software Architecture}

The robot controller is implemented in the Robot Operating System (ROS). ROS is not an operating system per se, but a framework for developing robotics applications. Figure 12 shows the nodelevel software architecture for the ROS implementation of the robotic enclave. Each node is implemented in Python. The software architecture is divided into multiple logical groupings.

ROS is divided into key functional groups which include nodes, topics, and services. Nodes are essentially encapsulations of logical functionality. Nodes communicate to other nodes through topics using a subscriber-publisher design pattern. Communications using topics are asynchronous (i.e., no blocking). Services exist as a means of synchronous communications between nodes. ROS services act like function calls and block the client when called. For the purpose of discussion, we will adopt the ROS taxonomy for objects within our software architecture.

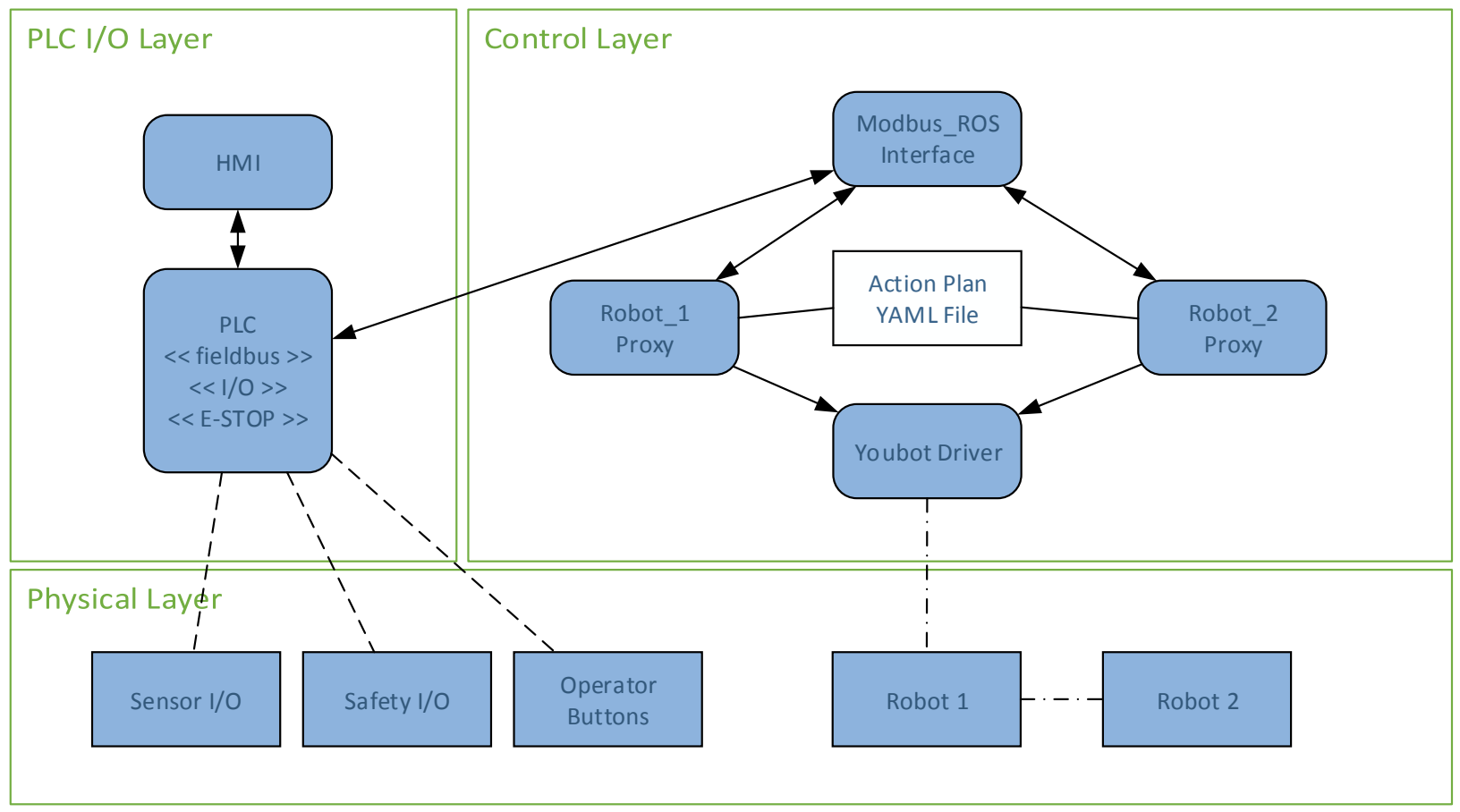

Figure 12. Robotics EnClaVe Node LeVel Software ArChitecture

\subsubsection{Control Layer}

The control layer is composed of nodes that provide control of the robots. The control layer includes the following functional nodes:

- Distributed controller nodes (Robot_1, Robot_2) that provide detailed control of the robots.

- YouBot driver to ROS interface node ${ }^{2}$.

${ }^{2}$ Code for the YouBot can be found at https://github.com/youbot. The Simple Open EtherCAT Master (SOEM) driver can be found at http://soem.berlios.de. 
- A Modbus interface for allowing any ROS node to monitor enclave sensors, operator buttons, and states from the PLC.

\subsubsection{PLC I/O Layer}

The PLCI/O layer serves as the bridge between ROS and the PLC. The PLC contains the supervisory control software of the enclave, which the ROS nodes monitor through the Modbus interface. As stated in 3.2.2, the emergency stop functionality is provided by the integrated safety PLC, and is activated by an emergency stop button and a light curtain attached to the enclave.

The HMI will serve as a graphical representation of the current states of the robots and the control system. The HMI will be developed using Python. The graphical user interface (GUI) will include controls, such as program start and stop, system state indicators, safety state indicators, and program selection.

\subsubsection{ROS Bags}

ROS provides a mechanism for recording and playing back ROS traffic called Bags. This feature may be useful for evaluating security performance related to replay attacks. ROS Bags are described in detail at http://wiki.ros.org/Bags. ROS Bags will also be very useful for replaying simulations for postmortem analysis.

\subsubsection{ROS versus ROS Industrial}

While ROS Industrial (ROS-I) provides many benefits to the developer and supports increasingly more industrial robots, currently no ROS-I support exists for the Kuka YouBot. Integration of the ROS driver into ROS-I is possible; however, it is unnecessary to meet the goals of the ICS cybersecurity project. It may be advantageous to transition to ROS-I in future releases of our robotics enclave to gain native support for PLCs, EtherCAT I/O, and other industrial protocols. Transitioning to the ROS-I framework will, at a minimum, require that the YouBot driver be encapsulated with a software wrapper that conforms to the essential driver interfaces described in the ROS-I Industrial Robot Driver Specification [5].

\subsubsection{Theory of Operation}

After initializing all required processes (e.g., ROS Core, YouBot driver, controllers, safety PLC, etc.), the robot controllers wait for the supervisory PLC's start/stop bit to be set. This is done via the operator buttons located on the front of the enclave. This bit is checked before each robot operation is initiated.

There are two machining stations available to each robot, as well as a robot-to-robot transfer in the middle of the table, and the queue. Parts move in a clockwise direction from the queue to each machining station, before being placed back into the queue.

The controller scans the current state of all machining stations on the enclave, which are provided by the PLC. The state of each machining station tells the controller whether: there is no part present, there is a part present and is currently being processed, or there is a part present and is ready for pick-up. Each machining station has a dedicated timer within the PLC which simulates the processing time for a part at the station to be "machined", which is started once the part is placed in the station. Once the timer has completed, the part is ready for pickup.

After scanning the current state of the machining stations, the controller will decide which operation it should complete from its available motions. There are only six required motions for the two robots with the current machining station setup (one of each type per robot): two for transferring a part 
to and from the queue, two for transferring the parts between stations, and two for the robot-to-robot transfer.

\subsubsection{Final Assembly}

A picture of the assembled robotics enclave is shown in Figure 13. The enclave includes a full 19" rack (left) that houses the PLC, safety controller, and industrial-grade router. The optical table (right) includes the two research-grade Kuka YouBot robots, machining pedestals, proximity sensors, control switches, and light curtain. Control of the enclave is actuated by the operator using the switch panel. A third station (not shown) includes a computer for the HMI, ROS master, and YouBot EtherCAT driver.

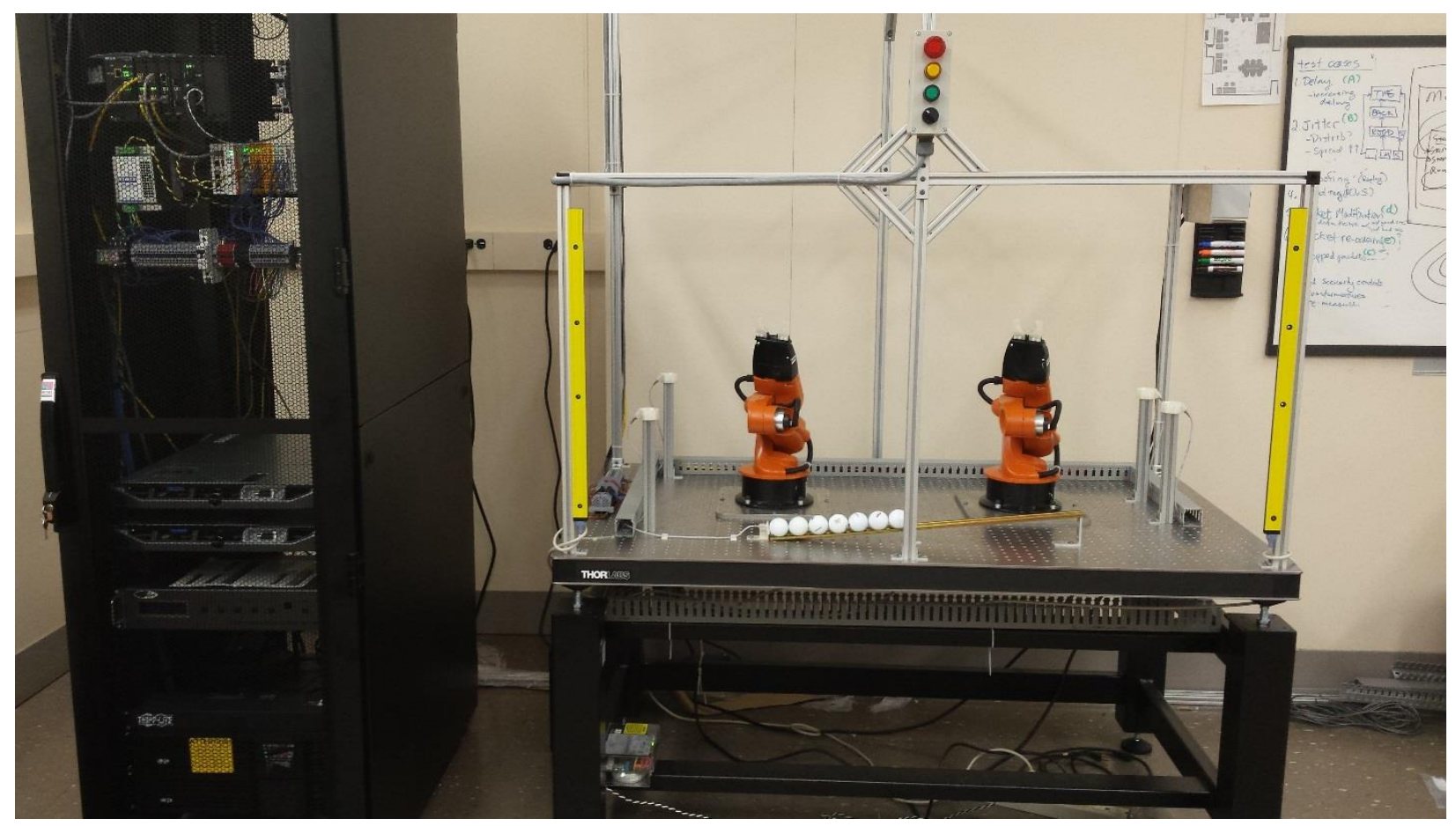

Figure 13. FULLY ASSEMBLED DISCRETE MANUFACTURING ROBOTICS ENCLAVE.

\subsection{Third Enclave Concepts}

\subsubsection{Intelligent Transportation System}

An intelligent transportation system that includes public infrastructure components, cooperative real-time embedded components, and wireless components are envisioned. The transportation system will be implemented by the Vanderbilt University Institute for Software Integrated Systems.

\subsubsection{Wide-area Networks with SCADA}

The concept of industrial control system cybersecurity is often connected with systems that employ Supervisory Control and Data Acquisition (SCADA) elements. Such systems operate as wide-area networks covering large geographical areas. SCADA systems may be simulated using the reconfigurable testbed currently allocated for the TE process or another enclave.

SCADA systems being considered for inclusion into the testbed include: 
1. A mass transit rail system in which control of the trains and infrastructure are conducted by a real-time SCADA system.

2. Liquid/Gas distribution systems that require real-time pressure control and monitoring, such as oil or natural gas pipelines and water treatment and distribution systems.

\subsection{Measurement Data Collection}

Measurement of packet flight is implemented as a separate enclave called the "Measurement Enclave." The measurement enclave serves two purposes. The first purpose of the enclave is to host the backbone switch and router for the cybersecurity lab. The second purpose is to host the computing resources necessary to capture and/or modify the packets as they traverse the network. As shown in Figure 16 in Appendix 8.2, all switches and diagnostics-capable computing or security devices are connected to the measurement rack. The switches in each of the enclaves are capable of port mirroring (i.e., SPAN porting), which enables mirroring of all traffic to a single port for packet capture. Each mirror port can be connected to the patch panel in the measurement rack. A performance-class server with multiple Ethernet interfaces is used to collect the packets transmitted by the replication ports.

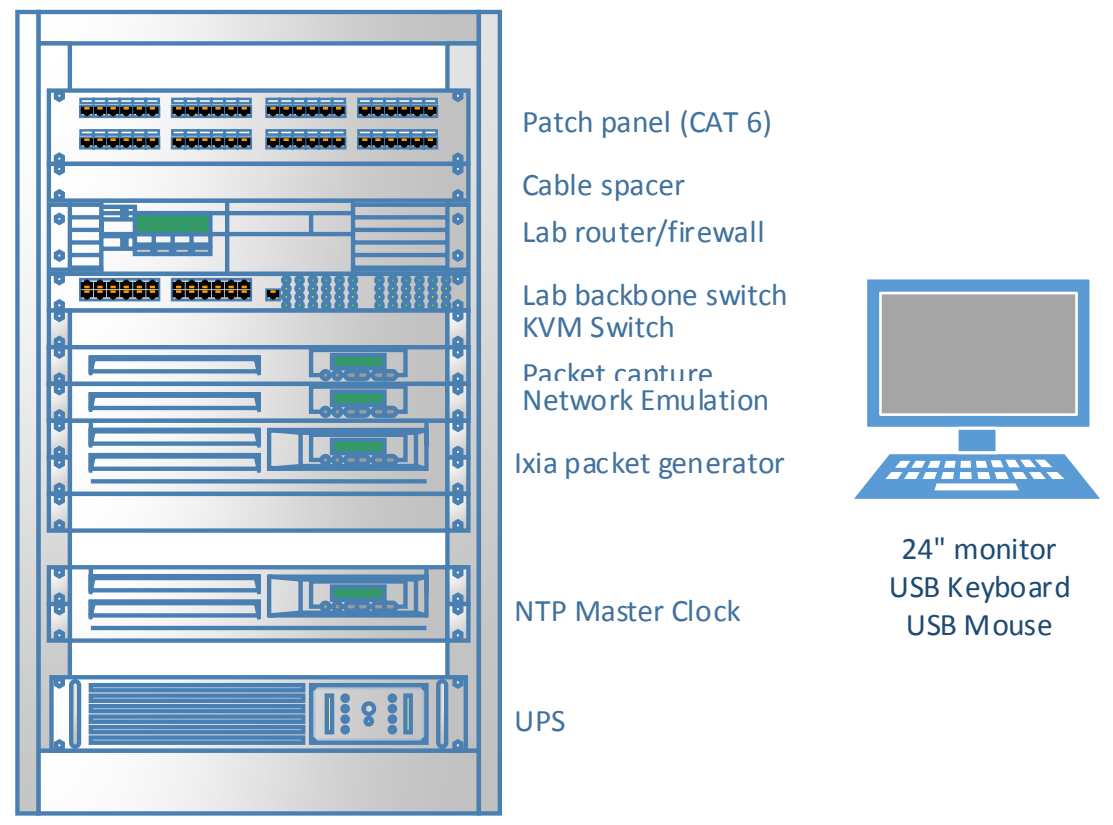

Figure 14. Measurement Enclave Rack Deployment

Wireshark and the Linux command-line program tcpdump are used to collect the packets and store the packets for offline analysis. An Ixia packet generator with Open Systems Interconnection (OSI) layer 3 through 7 capabilities is included in the rack for use cases that involve arbitrary packet generation. Packets captured in Wireshark can be replayed using the Ixia packet generator and may be useful for replay attacks and similar scenarios.

The Network Emulation server hosts the Ubuntu 12.04 Linux operating system. Many tools exist to provide the research team with network emulation tools and testing tools. Examples of these tools are listed in Table 1. 
In order to synchronize the time across all the machines in the enclaves, the measurement rack also includes a LANTIME M900 Network Time Protocol (NTP) timeserver. Having all of the machines source with this one master clock allows for accurate timestamping of logs and network captures across multiple machines. The offset and jitter of each machine to the master clock is logged in order to track and correct for time discrepancies between the machine logs.

TABle 1. Traffic Manipulation Tools for LinUX

\begin{tabular}{|l|l|}
\hline Tool & Description \\
\hline Ixia Anue & $\begin{array}{l}\text { Commercial-grade traffic shaping appliance for network device/system } \\
\text { testing. }\end{array}$ \\
\hline netem & $\begin{array}{l}\text { netem provides Network Emulation functionality for testing protocols by } \\
\text { emulating the properties of wide area networks. The current version } \\
\text { emulates variable delay, loss, duplication, and re-ordering. } \\
\text { http://www.linuxfoundation.org/collaborate/workgroups/networking/netem }\end{array}$ \\
\hline $\begin{array}{l}\text { Netem } \\
\text { (MasterShaper) }\end{array}$ & $\begin{array}{l}\text { MasterShaper is a network traffic shaper which provides a Web Interface for } \\
\text { Quality of Service functions of newer Linux 2.4- and 2.6- kernels. } \\
\text { http://www.mastershaper.org/ }\end{array}$ \\
\hline tc & A traffic control utility \\
\hline pf & A firewall with state-aware packet filtering \\
\hline
\end{tabular}

\subsection{Attack Computers}

Separate computers can be used for developing attack vectors on the various industrial processes described in this document. Computing resources that will be used for penetration testing are described in the following sections.

\subsubsection{Laptop Computer}

A laptop PC will be used as the primary platform for executing penetration tests. The software tool Metasploit (http://www.metasploit.com/) will be leveraged to execute penetration tests on the simulated processes.

\subsubsection{Ixia Traffic Generator}

The Ixia M2 can be leveraged for executing denial of service (DoS) and replay attacks on the simulated networks. Denial of service attacks are typically executed on networking equipment exposed to the internet; however, internally generated attacks are possible and will be investigated.

\subsubsection{Traffic Manipulation Server}

The effect of traffic manipulation attacks such as man-in-the-middle (MITM) attacks and network controls are simulated using an Ixia Anue appliance in the measurement enclave. 


\subsubsection{ROS Replay using Bags}

As described in 3.2.5.3, ROS Bags can be used to capture and replay ROS commands. This may be a useful tool for generating DoS and replay attacks. ROS Bag replays can be conducted from any of the machines that support ROS.

\section{Performance Metrics}

Rating the performance of an industrial control system is a challenging exercise. While industrial processes can be classified into general categories, no one set of metrics can be designed to cover all possible scenarios. Even identical designs may be implemented with different sensors, actuators, and control hardware. Top-level categories of processes include continuous processes, discrete processes, and a hybrid of continuous and discrete processes. Continuous processes are those in which materials flow through a system without pause or wait states. Discrete processes include those in which materials flow in quantized bundles and pauses or wait states are frequent. Many processes which appear to be mostly continuous are actually continuous processes with discrete elements and may be classified as hybrid processes. Process categories and examples of each are given in Table 2.

Table 2. Categories of Industrial Processes

\begin{tabular}{|l|l|}
\hline Category & Examples of processes \\
\hline Highly continuous process & Chemical manufacture \\
& Oil and Gas refineries \\
& Oil and Gas production and distribution \\
& Semiconductor manufacture \\
& Smelting \\
& Disinfection \\
\hline Highly discrete process & Robotic sorting \& assembly \\
& Automotive assembly \\
& Building automation \\
\hline Hybrid Continuous \& & Candy manufacture \\
Discrete & Pharmaceutical manufacture \\
& Metal-alloy manufacture \\
\hline
\end{tabular}

A one size fits all approach to a data-based assessment of performance of an industrial control system is very difficult and somewhat impractical. Much effort has been spent in identifying the technical indicators for assessing process performance. Both security metrics and process performance metrics exist and may be applied to industrial processes. Process performance metrics may include throughput, product quality, product error rate, and operational cost. Security metrics are well-defined for information technology in publications such as NIST SP 800-55 [4] and The Common Criteria for Information Technology Security Evaluation (CC) [6].

For the purpose of assessing the impact security has on process performance, it is necessary to measure the operational performance of the process. It makes little sense to measure security performance without first understanding how security technologies impact the performance of the process being protected. Therefore, for the purpose of assessing process performance, our approach is 
to focus on the technical performance indicators of the processes rather than information security metrics. Key metrics that will be analyzed are listed in Table 4 through Table 8 in the Appendix.

\section{ISA-99 Security}

The ICS Security Testbed will be used to validate the technical security requirements described in ISA/IEC-62443 (formerly ISA99), which mirrors the principles stated in NIST 800-82. This IEC series of documents is organized into groups shown in Figure 15. The 1-X series documents describe the purpose of the standard and establish a context in which the standard is used. The 2-X series documents describe the requirements of and how to implement policies and procedures of an ICS security program. The 3-X series documents present the architectural requirements for systems integrators and provides guidance on available security technologies that may be useful to a ICS integrator. The 4-X series focuses on requirements that component manufacturers must implement in their products to provide the functional hooks for a more secure implementation. Initial efforts will focus primarily on the requirements specified in ISA/IEC-62443-3-3.

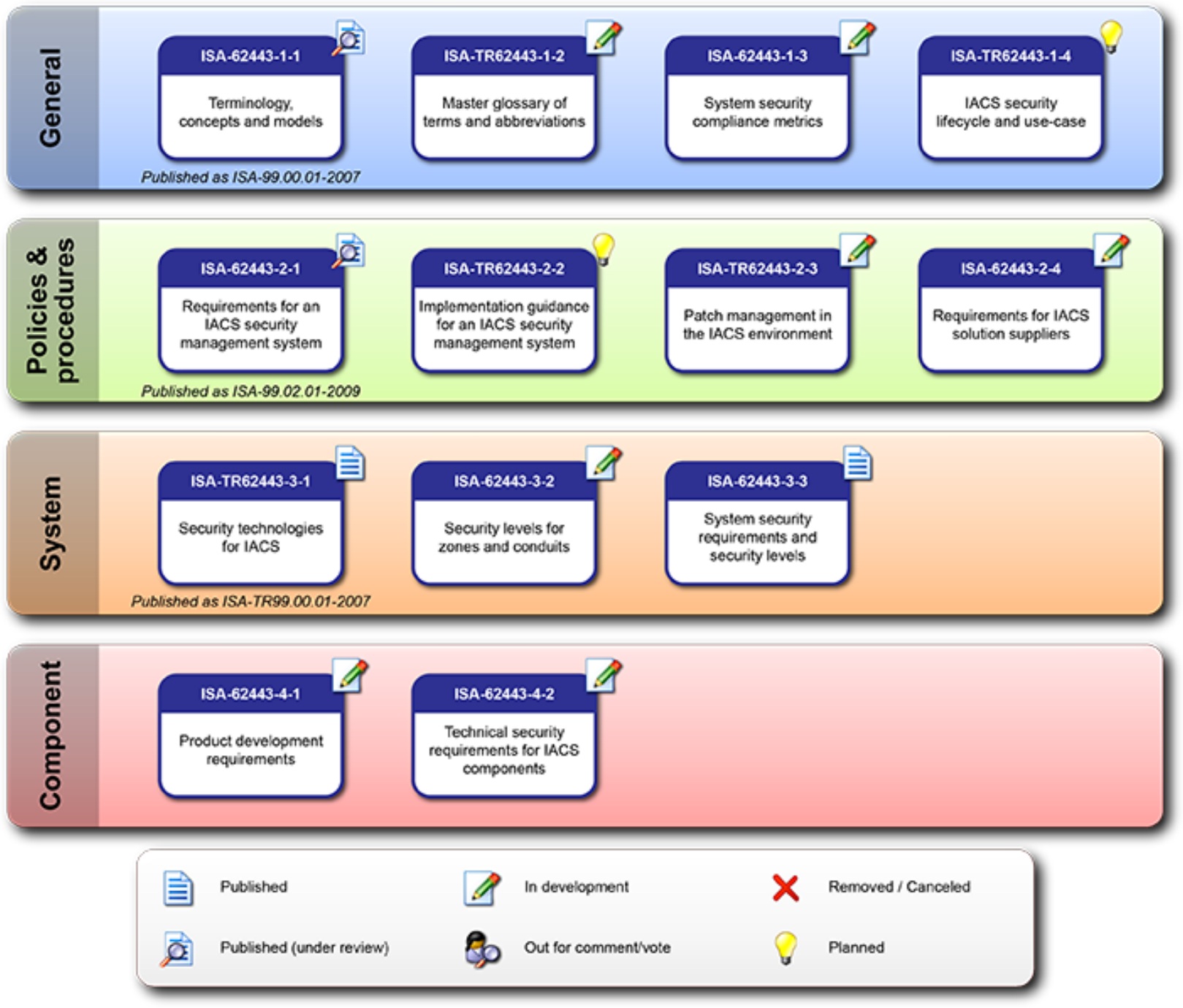

FiguRE 15. ISA/IEC-62443 ORGANIZATION OF STANDARDS DOCUMENTS 
The requirements specified in the 3-3 document are listed in Table 9 of the Appendix. A living document will be maintained in Microsoft Excel that documents the mapping of security requirements to use cases and technologies applied.

\section{Conclusions}

The NIST Industrial Control Systems Cybersecurity Testbed will provide an outstanding opportunity for the validation of existing security guidelines and standards as they pertain to critical infrastructure, such as public works systems, critical manufacturing systems, and other industrial control systems. By dividing the testbed into functional enclaves that represent multiple industrial interests, the testbed will serve as a platform for government, academic, and commercial researchers to experiment with security technologies and high assurance designs that make those systems more resilient to security threats, such as malicious cyber-attacks, user errors, and common network failures. The research produced from the use of the testbed will support the efforts of industry and government to develop more secure industrial control systems as well as improve the security of existing infrastructure.

\section{Bibliography}

[1] K. Stouffer, V. Pillitteri, S. Lightman, M. Abrams, and A. Hahn, "NIST Special Publication 800-82: Guide to Industrial Control Systems (ICS) Security," Gaithersburg, MD, Jun. 2015.

[2] J. J. Downs and E. F. Vogel, "A Plant-wide Industrial Problem Process," Comput. Chem. Eng., vol. 17, no. 3, pp. 245-255, 1993.

[3] A. a Cárdenas, S. Amin, Z.-S. Lin, Y.-L. Huang, C.-Y. Huang, and S. Sastry, "Attacks against process control systems: Risk assessment, detection, and response," Proc. 6th ACM Symp. Information, Comput. Commun. Secur., pp. 355-366, 2011.

[4] R. Candell, K. Stouffer, and D. Anand, "A Cybersecurity Testbed for Industrial Control Systems," in Proceedings of the 2014 Process Control and Safety Symposium, 2014.

[5] "ROS Industrial Driver Specification." [Online]. Available: http://wiki.ros.org/Industrial/Industrial_Robot_Driver_Spec.

[6] Ccra, "Common Criteria for Information Technology Security Evaluation Part 3 : Security assurance components," Security, no. September, pp. 1-321, 2012.

[7] A. Ordys, D. Uduehi, and M. A. Johnson, Eds., Process Control Performance Assessment: From Theory to Implementation (Advances in Industrial Control). Springer, 2010.

[8] Rachad Baroudi, KPI Mega Library: 17,000 Key Performance Indicators. Scotts Valley, California: CreateSpace Independent Publishing Platform, 2010. 
22 of 49 


\section{Appendix}

\subsection{Glossary}

Many acronyms and abbreviations are used throughout this document. Table 3 lists the common terms used repeatedly throughout the document.

TABLE 3. LIST OF TERMS

\begin{tabular}{|c|c|}
\hline Term & Definition \\
\hline $\mathrm{AC}$ & Alternating Current \\
\hline CIP & Common Industrial Protocol \\
\hline CPU & Central processing unit \\
\hline DC & Direct Current \\
\hline $\mathrm{DMZ}$ & Demilitarized Zone \\
\hline $\mathrm{GbE}$ & Gigabit Ethernet \\
\hline GE & Gilbert-Elliot \\
\hline GPS & Global Positioning System \\
\hline HDMI & High-Definition Multimedia Interface \\
\hline $\mathrm{HMI}$ & Human Machine Interface \\
\hline $\mathrm{Hz}$ & Hertz \\
\hline IGMP & Internet Group Management Protocol \\
\hline IP & Internet Protocol \\
\hline KVM & Keyboard, video, and mouse \\
\hline LAN & Local Area Network \\
\hline LED & Light emitting diode \\
\hline LSM & Loadable Software Module \\
\hline MST & Minimum Spanning Tree \\
\hline NIC & Network interface card \\
\hline OPC & OLE for Process Control \\
\hline $\mathrm{P} / \mathrm{S}$ & Power Supply \\
\hline PC & Personal computer \\
\hline $\mathrm{PCl}$ & Peripheral Component Interconnect \\
\hline PCle & Peripheral Component Interconnect Express \\
\hline PLC & Programmable Logic Controller \\
\hline Qos & Quality of Service \\
\hline RAM & Random Access Memory \\
\hline RPM & Revolutions per minute \\
\hline RSTP & Rapid Spanning Tree Protocol \\
\hline SATA & Serial ATA (Advanced Technology Attachment) \\
\hline STP & Spanning Tree Protocol \\
\hline TCP & Transmission Control Protocol \\
\hline TE & Tennessee Eastman \\
\hline UPS & Uninterruptable Power Supply \\
\hline USB & Universal Serial Bus \\
\hline V & Volt \\
\hline VGA & Video Graphics Array \\
\hline VLAN & Virtual Local Area Network \\
\hline
\end{tabular}




\begin{tabular}{|l|l|}
\hline Term & Definition \\
\hline$W$ & Watt(s) \\
\hline
\end{tabular}

\begin{tabular}{l|l} 
W & Watt(s)
\end{tabular} 
8.2 Testbed Network Architecture

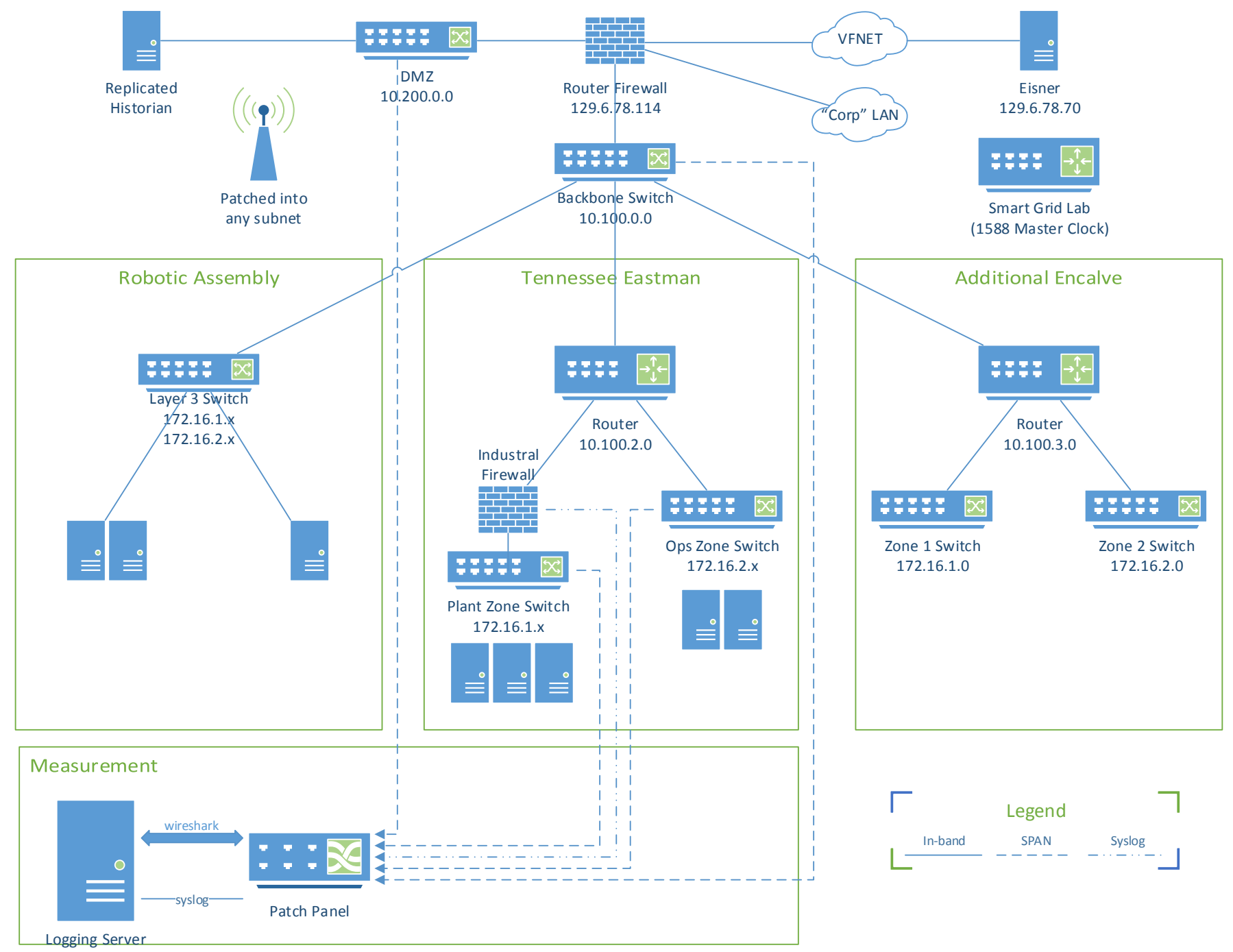

Figure 16. System Context for the Tennessee Eastman Enclave 


\subsection{Industrial Control Metrics}

Industrial metrics are listed in Table 4 through Table 8. Metrics for continuous processes, discrete processes, host performance, and network performance are organized into separate tables. The metrics presented here were selected through study of [7] and [8], and may be considered a small subset of a large number of different key performance indicators used in manufacturing and other industrial processes. Each organization must select performance indicators that are meaningful to their own operation.

Table 4. Performance Metrics for Continuous Processes

\begin{tabular}{|c|c|}
\hline Metric & Description \\
\hline$\%$ Process Availability & The ratio of process up-time to the sum of process up-time and down-time \\
\hline Product Quality & Statistical measures of product goodness or purity \\
\hline Process Variability & $\begin{array}{l}\text { Statistical measurement of how much a process variable deviates or } \\
\text { oscillates from its steady state value or set point. }\end{array}$ \\
\hline Steady State Error & $\begin{array}{l}\text { Oscillation over variability about a pre-determined set point and weights } \\
\text { both transient and steady state responses equally. }\end{array}$ \\
\hline Response Time & $\begin{array}{l}\text { A quantitative measurement of time to respond to a perturbation, such as a } \\
\text { step stimulus. }\end{array}$ \\
\hline Cost & The economic cost for running the process measured in currency \\
\hline Safety Margin & $\begin{array}{l}\text { Time taken to shutdown process after fault detection. This may be } \\
\text { particularly important where human safety is concerned. }\end{array}$ \\
\hline $\begin{array}{l}\text { \% Time Actuation at } \\
\text { Limits }\end{array}$ & $\begin{array}{l}\text { Measure of the amount of time a process control variable remains at a hard } \\
\text { limit. A common example of such a limit includes valves at full open or full } \\
\text { close. }\end{array}$ \\
\hline $\begin{array}{l}\text { Integrated Absolute } \\
\text { Error (IAE) }\end{array}$ & $\begin{array}{l}\text { Commonly used metric for evaluating the performance of a feedback } \\
\text { control loop. }\end{array}$ \\
\hline $\begin{array}{l}\text { Integrated Time- } \\
\text { weighted Absolute } \\
\text { Error (ITAE) }\end{array}$ & $\begin{array}{l}\text { Commonly used metric for evaluating the performance of a feedback } \\
\text { control loop. This particular metric weights the steady state error more } \\
\text { than the error introduced by the transient response. }\end{array}$ \\
\hline
\end{tabular}

Table 5. Performance Metrics for Discrete Processes

\begin{tabular}{|l|l|}
\hline Metric & Description \\
\hline Product Quality & A quantitative measurement of product goodness or purity \\
\hline Defect Rate & $\begin{array}{l}\text { Rate at which a product fails quality control checks due to errors in the } \\
\text { manufacturing process. }\end{array}$ \\
\hline Defects per unit & Statistical measures of the number of defects per unit \\
\hline Process Restart Rate & Number of times a process must be restarted in a given time interval. \\
\hline
\end{tabular}

\footnotetext{
${ }^{3}$ Not all process state variables have pre-determined set points. Manual overrides are available in some control systems.
} 


\begin{tabular}{|l|l|}
\hline Metric & Description \\
\hline $\begin{array}{l}\text { Variability of On-time } \\
\text { Actuation }\end{array}$ & Statistical measure of time between command and actuation completion. \\
\hline Process Duration & $\begin{array}{l}\text { Length of time to complete a sequence of tasks, such as a series of } \\
\text { assembly tasks in a robotic assembly system. }\end{array}$ \\
\hline
\end{tabular}

Table 6. Metrics for Measuring System Performance

\begin{tabular}{|l|l|}
\hline Metric & Description \\
\hline Volatile Memory & $\begin{array}{l}\text { Utilization of system memory typically reported as a percentage of total } \\
\text { RAM }\end{array}$ \\
\hline Non-volatile Memory & $\begin{array}{l}\text { Utilization of system memory typically reported as a percentage of total } \\
\text { system disk space }\end{array}$ \\
\hline CPU Utilization & Percentage of the total CPU usage time \\
\hline I/O Read Load & Total bytes read in the CPU I/O channel \\
\hline I/O Write Load & Total bytes written in the CPU I/O channel \\
\hline Missed Scans (Rate) & $\begin{array}{l}\text { When using a device such as a PLC that scans all variables before executing } \\
\text { the next iteration of control, the total number of sensor readings missed in } \\
\text { a given time interval. }\end{array}$ \\
\hline
\end{tabular}

Table 7. Nominal System Properties for Measuring System Performance

\begin{tabular}{|c|c|}
\hline Metric & Description \\
\hline Medium Type & $\begin{array}{l}\text { Examples include Copper, Fiber, Wireless and the associated protocol used } \\
\text { such as CAT-6 copper or } 802.11 \mathrm{~g} \text { wireless. }\end{array}$ \\
\hline $\begin{array}{l}\text { Physical Channel } \\
\text { Bandwidth }\end{array}$ & $\begin{array}{l}\text { The full bandwidth allocated to the channel. This can be useful for wireless } \\
\text { channels such as IEEE } 802.15 .4 \text { and modulated wired channels such as } \\
\text { Ethernet. }\end{array}$ \\
\hline $\begin{array}{l}\text { Rated Channel } \\
\text { Capacity }\end{array}$ & Rated capacity for transmitting and receiving elements in the network \\
\hline Channel Encoding & $\begin{array}{l}\text { Algorithm or structure used to encode the transmissions to include } \\
\text { interleaving, channel coding, modulation, and interference handling } \\
\text { properties }\end{array}$ \\
\hline $\begin{array}{l}\text { Environmental } \\
\text { Characteristics }\end{array}$ & $\begin{array}{l}\text { Mechanical, electrical, and electromagnetic properties of the environment } \\
\text { in which the system is deployed. }\end{array}$ \\
\hline Channel Compression & The data compression algorithm used for transmission \\
\hline $\begin{array}{l}\text { Rated Channel } \\
\text { Throughput }\end{array}$ & $\begin{array}{l}\text { The advertised theoretical throughput for a given transmitting or receiving } \\
\text { device }\end{array}$ \\
\hline $\begin{array}{l}\text { Routing Algorithms } \\
\text { Used }\end{array}$ & $\begin{array}{l}\text { The type of routing algorithm employed. Knowing the routing algorithm is } \\
\text { particularly useful for mobile ad-hoc networks and fully loaded ad-hoc } \\
\text { networks. }\end{array}$ \\
\hline
\end{tabular}




\begin{tabular}{|l|l|}
\hline Metric & Description \\
\hline $\begin{array}{l}\text { Switching Algorithms } \\
\text { Used }\end{array}$ & The type of layer 2 switching algorithm employed. \\
\hline $\begin{array}{l}\text { Determinism } \\
\text { Boundaries }\end{array}$ & Real-time constraints of the system which is known a priori \\
\hline
\end{tabular}

Table 8. Metrics for Measuring Network Performance

\begin{tabular}{|c|c|}
\hline Metric & Description \\
\hline Information Packet Rate & $\begin{array}{l}\text { Rate of information packet flow that is useful to the application } \\
\text { measured at the highest observable network layer. }\end{array}$ \\
\hline Information Bit Rate & $\begin{array}{l}\text { Rate of information bit flow that is useful to the application } \\
\text { measured at the highest observable network layer. }\end{array}$ \\
\hline Raw Packet Rate & Measured at layer 2 and includes overhead and retries \\
\hline Raw Bit Rate & Measured at layer 2 and includes overhead and retries \\
\hline Message Delay (Distribution) & $\begin{array}{l}\text { The delay for full messages (multiple packets) to be propagated } \\
\text { through the network or network link. Used for long packets } \\
\text { measured at the layer in which transport layer packets are } \\
\text { reassembled which is usually the application layer. }\end{array}$ \\
\hline Packet Delay (Distribution) & $\begin{array}{l}\text { The delay for single packets to be propagated through the network } \\
\text { or network link. }\end{array}$ \\
\hline Packet Delay Jitter & Variation in delay measured over an ensemble of packets. \\
\hline Processing Delay & $\begin{array}{l}\text { Delay introduced by network interconnect devices such as switches } \\
\text { and routers }\end{array}$ \\
\hline Queuing Delay & $\begin{array}{l}\text { Amount of time a packet spending in the input queue before being } \\
\text { processed }\end{array}$ \\
\hline Propagation Delay & $\begin{array}{l}\text { The amount of time a quanta of information takes to travel between } \\
\text { transmitter and receiver }{ }^{4}\end{array}$ \\
\hline Packet Collisions & Number of collisions typically reported by layer 2 devices \\
\hline Packet error rate & Rate of packet errors measured at the transport layer \\
\hline Packet loss rate & Rate of packet loss measured at the transport layer ${ }^{5}$ \\
\hline Packet Size (Distribution) & Distribution of the size of packets transmitted across the network. \\
\hline $\begin{array}{l}\text { Measured Determinism } \\
\text { Boundaries }\end{array}$ & Measured points of real-time determinism failure \\
\hline
\end{tabular}

\footnotetext{
${ }^{4}$ This may be particularly useful for wireless channels such as low earth orbital and geostationary satellite links in which the distance between transmitter and receiver is large relative to the transmission speed of the medium. ${ }^{5}$ Packet loss occurs due to collisions for non-reliable protocols and queuing loss due to network congestion.
} 


\subsection{NIST-SP 800-82 Security Overlay}

NIST Special Publication $800-82$ provides guidance in the application of security to industrial control systems. Rev 2 of NIST 800-82 Appendix G provides an overlay of NIST 800-53 security controls (requirements) to the industrial control systems. The risk management process and security controls overlay of 800-82 will be applied to the scenarios described in this report.

\subsection{ISA/IEC-62443-3-3 Security Requirements}

The security requirements in the ISA/IEC-62443 series of documents follow a security level vector approach. Increasing security levels represent an increase in perceived or calculated risk if the system integrity is compromised.

TABle 9. ISA/IEC-62443-3-3 RequiRements to SeCURITY LeVEL MAPPING ${ }^{6}$

\begin{tabular}{|c|c|c|c|c|}
\hline SRs and REs & SL 1 & SL 2 & SL 3 & SL 4 \\
\hline \multicolumn{5}{|l|}{ FR 1 - Identification and authentication control (IAC) } \\
\hline $\begin{array}{l}\text { SR } 1.1 \text { - Human user identification and } \\
\text { authentication }\end{array}$ & $\mathrm{x}$ & $\mathrm{x}$ & $\mathrm{X}$ & $x$ \\
\hline RE (1) Unique identification and authentication & & $\mathrm{x}$ & $\mathrm{x}$ & $\mathrm{x}$ \\
\hline $\begin{array}{l}\text { RE (2) Multifactor authentication for untrusted } \\
\text { networks }\end{array}$ & & & $\mathrm{x}$ & $x$ \\
\hline RE (3) Multifactor authentication for all networks & & & & $\mathrm{X}$ \\
\hline $\begin{array}{l}\text { SR } 1.2 \text { - Software process and device identification } \\
\text { and authentication }\end{array}$ & & $\mathrm{x}$ & $\mathrm{x}$ & $\mathrm{x}$ \\
\hline RE (1) Unique identification and authentication & & & $\mathrm{X}$ & $\mathrm{X}$ \\
\hline SR 1.3-Account management & $\mathrm{X}$ & $\mathrm{X}$ & $\mathrm{X}$ & $\mathrm{X}$ \\
\hline RE (1) Unified account management & & & $\mathrm{X}$ & $\mathrm{X}$ \\
\hline SR 1.4 - Identifier management & $\mathrm{X}$ & $\mathrm{X}$ & $\mathrm{X}$ & $\mathrm{X}$ \\
\hline SR 1.5 - Authenticator management & $\mathrm{X}$ & $\mathrm{X}$ & $\mathrm{X}$ & $\mathrm{X}$ \\
\hline $\begin{array}{l}\text { RE (1) Hardware security for software process } \\
\text { identity credentials }\end{array}$ & & & $x$ & $\mathrm{x}$ \\
\hline SR 1.6 - Wireless access management & $x$ & $\mathrm{X}$ & $\mathrm{X}$ & $\mathrm{X}$ \\
\hline RE (1) Unique identification and authentication & & $\mathrm{x}$ & $\mathrm{x}$ & $\mathrm{x}$ \\
\hline SR 1.7 - Strength of password-based authentication & $\mathrm{x}$ & $\mathrm{x}$ & $\mathrm{x}$ & $x$ \\
\hline
\end{tabular}

${ }^{6}$ This table was reproduced from IEC-62443-3-3 Draft 4, Annex B Table B-1. 


\begin{tabular}{|c|c|c|c|c|}
\hline SRs and REs & SL 1 & SL 2 & SL 3 & SL 4 \\
\hline $\begin{array}{l}\text { RE (1) Password generation and lifetime restrictions } \\
\text { for human users }\end{array}$ & & & $\mathrm{x}$ & $x$ \\
\hline RE (2) Password lifetime restrictions for all users & & & & $\mathrm{X}$ \\
\hline SR 1.8 - Public key infrastructure certificates & & $\mathrm{X}$ & $\mathrm{X}$ & $\mathrm{X}$ \\
\hline SR 1.9 - Strength of public key authentication & & $\mathrm{x}$ & $\mathrm{x}$ & $\mathrm{x}$ \\
\hline $\begin{array}{l}\text { RE (1) Hardware security for public key } \\
\text { authentication }\end{array}$ & & & $\mathrm{X}$ & $\mathrm{x}$ \\
\hline SR 1.10 - Authenticator feedback & $\mathrm{X}$ & $\mathrm{x}$ & $x$ & $x$ \\
\hline SR 1.11 - Unsuccessful login attempts & $\mathrm{X}$ & $\mathrm{X}$ & $\mathrm{X}$ & $\mathrm{x}$ \\
\hline SR 1.12 - System use notification & $\mathrm{X}$ & $x$ & $\mathrm{X}$ & $\mathrm{X}$ \\
\hline SR 1.13 - Access via untrusted networks & $\mathrm{X}$ & $\mathrm{X}$ & $\mathrm{X}$ & $\mathrm{X}$ \\
\hline RE (1)Explicit access request approval & & $\mathrm{x}$ & $\mathrm{X}$ & $\mathrm{X}$ \\
\hline \multicolumn{5}{|l|}{ FR 2 - Use control (UC) } \\
\hline SR 2.1 - Authorization enforcement & $\mathrm{X}$ & $\mathrm{x}$ & $\mathrm{x}$ & $\mathrm{x}$ \\
\hline RE (1) Authorization enforcement for all users & & $\mathrm{X}$ & $x$ & $x$ \\
\hline RE (2) Permission mapping to roles & & $\mathrm{x}$ & $\mathrm{x}$ & $x$ \\
\hline RE (3) Supervisor override & & & $\mathrm{x}$ & $\mathrm{x}$ \\
\hline RE (4) Dual approval & & & & $\mathrm{x}$ \\
\hline SR 2.2 - Wireless use control & $\mathrm{x}$ & $\mathrm{x}$ & $\mathrm{x}$ & $\mathrm{X}$ \\
\hline $\begin{array}{l}\text { RE (1) Identify and report unauthorized wireless } \\
\text { devices }\end{array}$ & & & $x$ & $x$ \\
\hline $\begin{array}{l}\text { SR } 2.3 \text { - Use control for portable and mobile } \\
\text { devices }\end{array}$ & $\mathrm{x}$ & $x$ & $x$ & $x$ \\
\hline $\begin{array}{l}\text { RE (1) Enforcement of security status of portable } \\
\text { and mobile devices }\end{array}$ & & & $x$ & $x$ \\
\hline SR 2.4-Mobile code & $\mathrm{x}$ & $\mathrm{x}$ & $\mathrm{x}$ & $x$ \\
\hline RE (1) Mobile code integrity check & & & $\mathrm{X}$ & $\mathrm{X}$ \\
\hline SR 2.5 - Session lock & $\mathrm{x}$ & $\mathrm{x}$ & $\mathrm{X}$ & $\mathrm{X}$ \\
\hline SR 2.6 - Remote session termination & & $\mathrm{X}$ & $\mathrm{X}$ & $\mathrm{x}$ \\
\hline
\end{tabular}




\begin{tabular}{|c|c|c|c|c|}
\hline SRs and REs & SL 1 & SL 2 & SL 3 & SL 4 \\
\hline SR 2.7 - Concurrent session control & & & $\mathrm{X}$ & $\mathrm{X}$ \\
\hline SR 2.8 - Auditable events & $\mathrm{X}$ & $\mathrm{X}$ & $\mathrm{X}$ & $\mathrm{X}$ \\
\hline RE (1) Centrally managed, system-wide audit trail & & & $X$ & $X$ \\
\hline SR 2.9 - Audit storage capacity & $\mathrm{X}$ & $X$ & $X$ & $\mathrm{x}$ \\
\hline $\begin{array}{l}\text { RE (1) Warn when audit record storage capacity } \\
\text { threshold reached }\end{array}$ & & & $\mathrm{x}$ & $\mathrm{x}$ \\
\hline SR 2.10 - Response to audit processing failures & $\mathrm{X}$ & $\mathrm{x}$ & $\mathrm{X}$ & $\mathrm{x}$ \\
\hline SR 2.11 - Timestamps & & $\mathrm{X}$ & $\mathrm{X}$ & $\mathrm{x}$ \\
\hline RE (1)Internal time synchronization & & & $X$ & $\mathrm{x}$ \\
\hline RE (2) Protection of time source integrity & & & & $\mathrm{x}$ \\
\hline SR 2.12 - Non-repudiation & & & $\mathrm{x}$ & $\mathrm{x}$ \\
\hline RE (1) Non-repudiation for all users & & & & $x$ \\
\hline \multicolumn{5}{|l|}{ FR 3 - System integrity (SI) } \\
\hline SR 3.1-Communication integrity & $\mathrm{x}$ & $\mathrm{x}$ & $\mathrm{x}$ & $\mathrm{x}$ \\
\hline RE (1) Cryptographic integrity protection & & & $\mathrm{X}$ & $\mathrm{x}$ \\
\hline SR 3.2 - Malicious code protection & $\mathrm{X}$ & $\mathrm{X}$ & $\mathrm{X}$ & $\mathrm{X}$ \\
\hline $\begin{array}{l}\text { RE (1) Malicious code protection on entry and exit } \\
\text { points }\end{array}$ & & $\mathrm{x}$ & $\mathrm{X}$ & $\mathrm{x}$ \\
\hline $\begin{array}{l}\text { RE (2) Central management and reporting for } \\
\text { malicious code protection }\end{array}$ & & & $\mathrm{x}$ & $x$ \\
\hline SR 3.3 - Security functionality verification & $\mathrm{X}$ & $\mathrm{x}$ & $x$ & $\mathrm{x}$ \\
\hline $\begin{array}{l}\text { RE (1) Automated mechanisms for security } \\
\text { functionality verification }\end{array}$ & & & $x$ & $x$ \\
\hline $\begin{array}{l}\text { RE (2) Security functionality verification during } \\
\text { normal operation }\end{array}$ & & & & $x$ \\
\hline SR 3.4 - Software and information integrity & $\mathrm{X}$ & $\mathrm{x}$ & $\mathrm{x}$ & $\mathrm{x}$ \\
\hline $\begin{array}{l}\text { RE (1) Automated notification about integrity } \\
\text { violations }\end{array}$ & & & $\mathrm{x}$ & $x$ \\
\hline SR 3.5 - Input validation & $\mathrm{X}$ & $\mathrm{X}$ & $\mathrm{X}$ & $\mathrm{X}$ \\
\hline
\end{tabular}




\begin{tabular}{|c|c|c|c|c|}
\hline SRs and REs & SL 1 & SL 2 & SL 3 & SL 4 \\
\hline SR 3.6 - Deterministic output & $\mathrm{X}$ & $\mathrm{X}$ & $\mathrm{X}$ & $\mathrm{x}$ \\
\hline SR 3.7 - Error handling & & $\mathrm{X}$ & $\mathrm{x}$ & $\mathrm{x}$ \\
\hline SR 3.8 - Session integrity & & $\mathrm{X}$ & $\mathrm{X}$ & $\mathrm{X}$ \\
\hline $\begin{array}{l}\text { RE (1) Invalidation of session IDs after session } \\
\text { termination }\end{array}$ & & & $x$ & $\mathrm{x}$ \\
\hline RE (2)Unique session ID generation & & & $\mathrm{x}$ & $\mathrm{X}$ \\
\hline RE (3) Randomness of session IDs & & & & $\mathrm{X}$ \\
\hline SR 3.9- Protection of audit information & & $\mathrm{x}$ & $\mathrm{X}$ & $\mathrm{X}$ \\
\hline RE (1) Audit records on write-once media & & & & $\mathrm{X}$ \\
\hline \multicolumn{5}{|l|}{ FR 4 - Data confidentiality (DC) } \\
\hline SR 4.1 - Information confidentiality & $x$ & $\mathrm{x}$ & $\mathrm{x}$ & $\mathrm{X}$ \\
\hline $\begin{array}{l}\text { RE (1) Protection of confidentiality at rest or in } \\
\text { transit via untrusted networks }\end{array}$ & & $x$ & $x$ & $\mathrm{x}$ \\
\hline $\begin{array}{l}\text { RE (2) Protection of confidentiality across zone } \\
\text { boundaries }\end{array}$ & & & & $\mathrm{x}$ \\
\hline SR 4.2 - Information persistence & & $x$ & $x$ & $\mathrm{x}$ \\
\hline RE (1) Purging of shared memory resources & & & $\mathrm{x}$ & $\mathrm{x}$ \\
\hline SR 4.3 - Use of cryptography & $x$ & $\mathrm{x}$ & $\mathrm{x}$ & $\mathrm{x}$ \\
\hline \multicolumn{5}{|l|}{ FR 5 - Restricted data flow (RDF) } \\
\hline SR 5.1- Network segmentation & $x$ & $\mathrm{x}$ & $\mathrm{x}$ & $\mathrm{X}$ \\
\hline RE (1) Physical network segmentation & & $x$ & $\mathrm{x}$ & $\mathrm{X}$ \\
\hline $\begin{array}{l}\text { RE (2) Independence from non-control system } \\
\text { networks }\end{array}$ & & & $x$ & $\mathrm{x}$ \\
\hline $\begin{array}{l}\text { RE (3) Logical and physical isolation of critical } \\
\text { networks }\end{array}$ & & & & $\mathrm{x}$ \\
\hline SR 5.2-Zone boundary protection & $x$ & $\mathrm{x}$ & $\mathrm{x}$ & $\mathrm{X}$ \\
\hline RE (1) Deny by default, allow by exception & & $x$ & $x$ & $x$ \\
\hline RE (2) Island mode & & & $\mathrm{X}$ & $x$ \\
\hline RE (3) Fail close & & & $X$ & $\mathrm{X}$ \\
\hline
\end{tabular}




\begin{tabular}{|c|c|c|c|c|}
\hline SRs and REs & SL 1 & SL 2 & SL 3 & SL 4 \\
\hline $\begin{array}{l}\text { SR 5.3-General purpose person-to-person } \\
\text { communication restrictions }\end{array}$ & $\mathrm{x}$ & $\mathrm{x}$ & $\mathrm{x}$ & $x$ \\
\hline $\begin{array}{l}\text { RE (1) Prohibit all general purpose person-to-person } \\
\text { communications }\end{array}$ & & & $x$ & $\mathrm{x}$ \\
\hline SR 5.4-Application partitioning & $x$ & $\mathrm{X}$ & $x$ & $\mathrm{X}$ \\
\hline \multicolumn{5}{|l|}{ FR 6 - Timely response to events (TRE) } \\
\hline SR 6.1 - Audit log accessibility & $x$ & $x$ & $\mathrm{x}$ & $\mathrm{x}$ \\
\hline RE (1) Programmatic access to audit logs & & & $\mathrm{X}$ & $\mathrm{x}$ \\
\hline SR 6.2 - Continuous monitoring & & $\mathrm{X}$ & $x$ & $\mathrm{x}$ \\
\hline \multicolumn{5}{|l|}{ FR 7-Resource availability (RA) } \\
\hline SR 7.1-Denial of service protection & $\mathrm{x}$ & $\mathrm{x}$ & $\mathrm{x}$ & $x$ \\
\hline RE (1) Manage communication loads & & $\mathrm{x}$ & $\mathrm{X}$ & $\mathrm{x}$ \\
\hline $\begin{array}{l}\text { RE (2) Limit DoS effects to other systems or } \\
\text { networks }\end{array}$ & & & $x$ & $x$ \\
\hline SR 7.2-Resource management & $\mathrm{x}$ & $\mathrm{x}$ & $\mathrm{X}$ & $x$ \\
\hline SR 7.3-Control system backup & $\mathrm{x}$ & $\mathrm{X}$ & $\mathrm{X}$ & $\mathrm{X}$ \\
\hline RE (1) Backup verification & & $x$ & $\mathrm{X}$ & $\mathrm{X}$ \\
\hline RE (2) Backup automation & & & $x$ & $x$ \\
\hline SR 7.4 - Control system recovery and reconstitution & $\mathrm{x}$ & $x$ & $x$ & $\mathrm{x}$ \\
\hline SR 7.5 - Emergency power & $\mathrm{x}$ & $\mathrm{x}$ & $x$ & $\mathrm{X}$ \\
\hline $\begin{array}{l}\text { SR } 7.6 \text { - Network and security configuration } \\
\text { settings }\end{array}$ & $\mathrm{x}$ & $\mathrm{x}$ & $x$ & $\mathrm{x}$ \\
\hline $\begin{array}{l}\text { RE (1) Machine-readable reporting of current } \\
\text { security settings }\end{array}$ & & & $\mathrm{X}$ & $x$ \\
\hline SR 7.7 - Least functionality & $\mathrm{x}$ & $\mathrm{x}$ & $\mathrm{X}$ & $\mathrm{X}$ \\
\hline SR 7.8 - Control system component inventory & & $\mathrm{x}$ & $\mathrm{X}$ & $\mathrm{X}$ \\
\hline
\end{tabular}




\subsection{Tennessee Eastman Simulation Case Study}

\subsubsection{Description of the Simulation}

The TE model was developed to foster innovation in controller design. The model is non-dynamic and can be considered zero-order; however, the process itself has many variables and can therefore become unpredictable. The model comes with twenty disturbance types defined by Downs and Vogel of which the first twelve were used when applied as one disturbance. To investigate the effect of a generic inline network device such as an industrial firewall on the performance of typical industrial process, a Simulink model was constructed using the TE plant and decentralized controller implemented by Ricker [2] and a model of a generic network security device. The architecture for the model is shown in Figure 17.

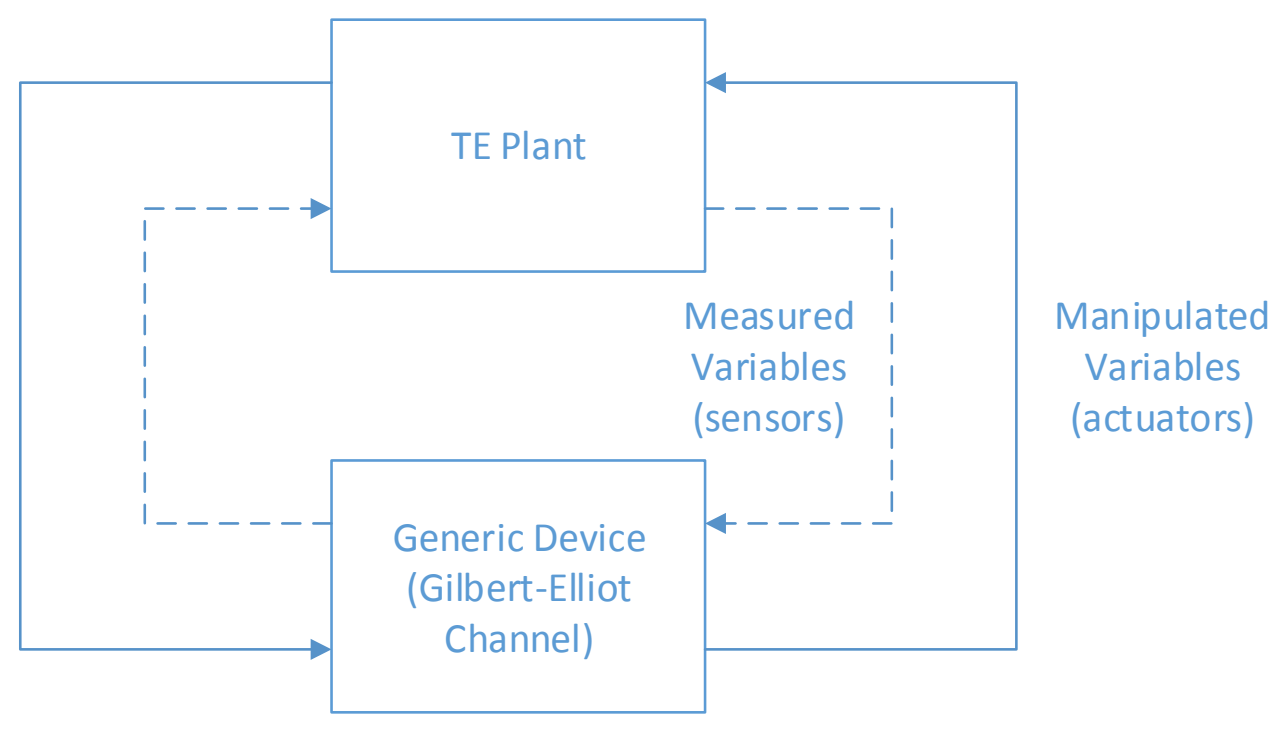

Figure 17. Tennessee Eastman Simulation ArChitecture using a Gilbert-Elliot Channel Model

A two-state Gilbert-Elliot (GE) model [5] was chosen to emulate the behavior of a network device in which a scanning device such as a PLC interrogates sensors within a plant process. [6] Each scan places a load on the device. The applied load depends on the number of devices scanned, the protocols used, the processing and memory capabilities of the device, and the complexity of the rules being applied. The latency distribution of packets between the plant and controller will determine what sensors are scanned and what actuators are updated successfully. It is assumed that the link states will follow a burst error pattern similar to a two-state Gilbert model that depends on the load applied. The model for a two-state GE channel is shown in Figure 18. ${ }^{7}$ Each measured variable is implemented with a Gilbert channel that is independent of the other measured variables; however, each channel is implemented with the same $P$ and $R$ values.

\footnotetext{
${ }^{7}$ The Gilbert model is one approach to characterizing a generic security device. Using a probabilistic channel model can provide clear guidance to component manufacturers on how to design their devices and to system integrators on how to deploy those devices. Research is required and industry participation will be necessary to select an appropriate channel model for industrial networks. A documented test approach for a generic industrial security device may be necessary to achieve industry acceptance.
} 
The GE model has two states, "good" and "bad." The good state indicates that the network device is loaded such that traffic is allowed to pass through in time to be captured by the scanning device within its scan interval. The bad state indicates that the network device is overloaded to the point that packets are delayed enough to be missed by the scan or discarded by the device. When the GE channel is in the bad state, the PLC will use the last known measured value.

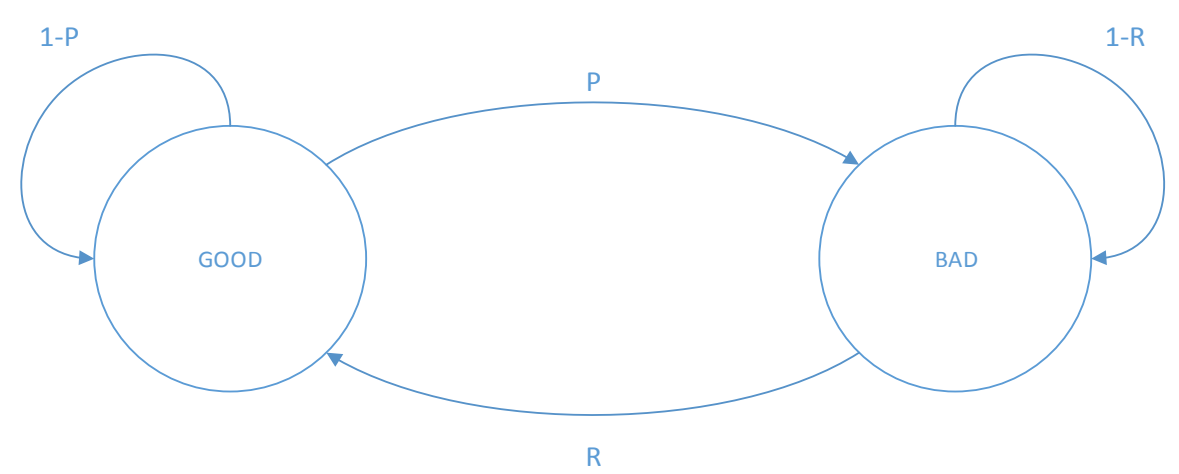

Figure 18. Simple Gilbert Channel Model for a Generic Network Device

\subsubsection{Discussion of the Results}

A parametric sweep was performed by varying the $P$ and $R$ probabilities for each disturbance type under steady state conditions in which set points did not vary during the disturbance. In addition, four multi-point disturbance vectors were activated at random, and the probabilities were again swept for each. For each disturbance type and probability pair, a time series was recorded for each measured process variable and quality indicator as a function of $P$ and $R$ probability values. The disturbance vector was applied at time $t=0$, and only the transient response was considered for each. The raw results were stored in a separate folder for each disturbance vector. ${ }^{8}$ The time series data was then post-processed to produce selected metrics. Computed metrics types for each measure variable and quality indicator are listed in Table 10. Metrics were stored in a Microsoft Access 2010 database allowing for filtering and analysis of the data.

Table 10. Metrics Types Collected from the TE Simulation

\begin{tabular}{|l|l|}
\hline Metric & Description \\
\hline Shutdown indicator & True if the plant shutdown \\
\hline Average deviation & $\begin{array}{l}\text { The average deviation detected from the baseline condition of all } \\
\text { measured variables }\end{array}$ \\
\hline Maximum deviation & $\begin{array}{l}\text { The maximum deviation detected from the baseline condition of all } \\
\text { measured variables }\end{array}$ \\
\hline Variance of deviation & $\begin{array}{l}\text { The variance of the deviation from the baseline case of all } \\
\text { measured variables }\end{array}$ \\
\hline
\end{tabular}

${ }^{8}$ The raw output data is stored in the GitHub repository in ZIP format. The TEsim code and data repository can be cloned from the URL https://github.com/NIST-ICS-SEC-TB/TEsim.git.

${ }^{9}$ A shutdown condition will occur in TEsim if a measured variable exceeds a predefined threshold. Shutdown thresholds are hard-coded in temexd.c and altering the thresholds requires a recompile. 


\begin{tabular}{|l|l|}
\hline Metric & Description \\
\hline Correlation to baseline & $\begin{array}{l}\text { The correlation of the measured variable to the baseline case of all } \\
\text { measured variables }\end{array}$ \\
\hline Operating Cost Correlation & The correlation between operating cost and the baseline case \\
\hline Operating Cost Max. Deviation & The maximum deviation of operating cost to the baseline case \\
\hline Percent G Correlation & The correlation of operating cost to the baseline case \\
\hline Percent G Max. Deviation & $\begin{array}{l}\text { The maximum deviation between operating cost and the baseline } \\
\text { case }\end{array}$ \\
\hline $\begin{array}{l}\text { Percent G Variance of } \\
\text { Deviation }\end{array}$ & The variance of the molar percentage of G from the baseline case \\
\hline
\end{tabular}

A full listing of the calculated metrics is given in Table 11.

TABle 11. Listing of the MEtRICS TABle in the Results DatABASE

\begin{tabular}{|l|l|}
\hline Name & Type \\
\hline$P$ & Double \\
\hline$R$ & Double \\
\hline IDVnum & Double \\
\hline Shutdown & Integer \\
\hline AVGDEV from A feed stream 1 & Double \\
\hline AVGDEV from D feed stream 2 & Double \\
\hline AVGDEV from E feed stream 3 & Double \\
\hline AVGDEV from A and C feed stream 4 & Double \\
\hline AVGDEV from Recycle flow Stream 8 & Double \\
\hline AVGDEV from Reactor feed rate stream 6 & Double \\
\hline AVGDEV from Reactor pressure & Double \\
\hline AVGDEV from Reactor level & Double \\
\hline AVGDEV from Reactor temperature & Double \\
\hline AVGDEV from Purge rate stream 9 & Double \\
\hline AVGDEV from Product separator temperature & Double \\
\hline AVGDEV from Product separator level & Double \\
\hline AVGDEV from Product separator pressure & Double \\
\hline AVGDEV from Product separator underflow stream 10 & Double \\
\hline AVGDEV from stripper level & Double \\
\hline AVGDEV from stripper pressure & Double \\
\hline AVGDEV from Stripper underflow stream 11 & Double \\
\hline AVGDEV from stripper temperature & Double \\
\hline AVGDEV from strippx steam dew & Double \\
\hline AVGDEV from Compressor work & Double \\
\hline AVGDEV from Reactor cooling water outlet temperature & Double \\
\hline AVGDEV from separator cooling water outlet temperature & Double \\
\hline AVGDEV from Reactor feed A & Double \\
\hline
\end{tabular}




\begin{tabular}{|c|c|}
\hline Name & Type \\
\hline AVGDEV from Reactor feed B & Double \\
\hline AVGDEV from Reactor feed C & Double \\
\hline AVGDEV from Reactor feed D & Double \\
\hline AVGDEV from Reactor feed $\mathrm{E}$ & Double \\
\hline AVGDEV from Reactor feed $\mathrm{F}$ & Double \\
\hline AVGDEV from Purge gas $A$ & Double \\
\hline AVGDEV from Purge gas B & Double \\
\hline AVGDEV from Purge gas $C$ & Double \\
\hline AVGDEV from Purge gas D & Double \\
\hline AVGDEV from Purge gas $\mathrm{E}$ & Double \\
\hline AVGDEV from Purge gas $F$ & Double \\
\hline AVGDEV from Purge gas $G$ & Double \\
\hline AVGDEV from Purge gas $\mathrm{H}$ & Double \\
\hline AVGDEV from Product D & Double \\
\hline AVGDEV from Product $\mathrm{E}$ & Double \\
\hline AVGDEV from Product $F$ & Double \\
\hline AVGDEV from Product G & Double \\
\hline AVGDEV from Product $\mathrm{H}$ & Double \\
\hline MAXDEV from A feed stream 1 & Double \\
\hline MAXDEV from D feed stream 2 & Double \\
\hline MAXDEV from E feed stream 3 & Double \\
\hline MAXDEV from $A$ and $C$ feed stream 4 & Double \\
\hline MAXDEV from Recycle flow Stream 8 & Double \\
\hline MAXDEV from Reactor feed rate stream 6 & Double \\
\hline MAXDEV from Reactor pressure & Double \\
\hline MAXDEV from Reactor level & Double \\
\hline MAXDEV from Reactor temperature & Double \\
\hline MAXDEV from Purge rate stream 9 & Double \\
\hline MAXDEV from Product separator temperature & Double \\
\hline MAXDEV from Product separator level & Double \\
\hline MAXDEV from Product separator pressure & Double \\
\hline MAXDEV from Product separator underflow stream 10 & Double \\
\hline MAXDEV from stripper level & Double \\
\hline MAXDEV from stripper pressure & Double \\
\hline MAXDEV from Stripper underflow stream 11 & Double \\
\hline MAXDEV from stripper temperature & Double \\
\hline MAXDEV from strippx steam dew & Double \\
\hline MAXDEV from Compressor work & Double \\
\hline MAXDEV from Reactor cooling water outlet temperature & Double \\
\hline MAXDEV from separator cooling water outlet temperature & Double \\
\hline MAXDEV from Reactor feed $A$ & Double \\
\hline MAXDEV from Reactor feed B & Double \\
\hline MAXDEV from Reactor feed C & Double \\
\hline MAXDEV from Reactor feed D & Double \\
\hline MAXDEV from Reactor feed $\mathrm{E}$ & Double \\
\hline
\end{tabular}




\begin{tabular}{|c|c|}
\hline Name & Type \\
\hline MAXDEV from Reactor feed $\mathrm{F}$ & Double \\
\hline MAXDEV from Purge gas $A$ & Double \\
\hline MAXDEV from Purge gas $B$ & Double \\
\hline MAXDEV from Purge gas $C$ & Double \\
\hline MAXDEV from Purge gas D & Double \\
\hline MAXDEV from Purge gas E & Double \\
\hline MAXDEV from Purge gas $F$ & Double \\
\hline MAXDEV from Purge gas $G$ & Double \\
\hline MAXDEV from Purge gas $\mathrm{H}$ & Double \\
\hline MAXDEV from Product D & Double \\
\hline MAXDEV from Product $E$ & Double \\
\hline MAXDEV from Product $F$ & Double \\
\hline MAXDEV from Product $\mathrm{G}$ & Double \\
\hline MAXDEV from Product $\mathrm{H}$ & Double \\
\hline VAR from $A$ feed stream 1 & Double \\
\hline VAR from $D$ feed stream 2 & Double \\
\hline VAR from $\mathrm{E}$ feed stream 3 & Double \\
\hline VAR from $A$ and $C$ feed stream 4 & Double \\
\hline VAR from Recycle flow Stream 8 & Double \\
\hline VAR from Reactor feed rate stream 6 & Double \\
\hline VAR from Reactor pressure & Double \\
\hline VAR from Reactor level & Double \\
\hline VAR from Reactor temperature & Double \\
\hline VAR from Purge rate stream 9 & Double \\
\hline VAR from Product separator temperature & Double \\
\hline VAR from Product separator level & Double \\
\hline VAR from Product separator pressure & Double \\
\hline VAR from Product separator underflow stream 10 & Double \\
\hline VAR from stripper level & Double \\
\hline VAR from stripper pressure & Double \\
\hline VAR from Stripper underflow stream 11 & Double \\
\hline VAR from stripper temperature & Double \\
\hline VAR from strippx steam dew & Double \\
\hline VAR from Compressor work & Double \\
\hline VAR from Reactor cooling water outlet temperature & Double \\
\hline VAR from separator cooling water outlet temperature & Double \\
\hline VAR from Reactor feed A & Double \\
\hline VAR from Reactor feed B & Double \\
\hline VAR from Reactor feed C & Double \\
\hline VAR from Reactor feed D & Double \\
\hline VAR from Reactor feed $\mathrm{E}$ & Double \\
\hline VAR from Reactor feed $\mathrm{F}$ & Double \\
\hline VAR from Purge gas $A$ & Double \\
\hline VAR from Purge gas B & Double \\
\hline VAR from Purge gas $C$ & Double \\
\hline
\end{tabular}




\begin{tabular}{|c|c|}
\hline Name & Type \\
\hline VAR from Purge gas D & Double \\
\hline VAR from Purge gas $E$ & Double \\
\hline VAR from Purge gas $\mathrm{F}$ & Double \\
\hline VAR from Purge gas $G$ & Double \\
\hline VAR from Purge gas $\mathrm{H}$ & Double \\
\hline VAR from Product D & Double \\
\hline VAR from Product $\mathrm{E}$ & Double \\
\hline VAR from Product $F$ & Double \\
\hline VAR from Product $\mathrm{G}$ & Double \\
\hline VAR from Product $\mathrm{H}$ & Double \\
\hline CORR of $A$ feed stream 1 & Double \\
\hline CORR of D feed stream 2 & Double \\
\hline CORR of E feed stream 3 & Double \\
\hline CORR of $A$ and $C$ feed stream 4 & Double \\
\hline CORR of Recycle flow Stream 8 & Double \\
\hline CORR of Reactor feed rate stream 6 & Double \\
\hline CORR of Reactor pressure & Double \\
\hline CORR of Reactor level & Double \\
\hline CORR of Reactor temperature & Double \\
\hline CORR of Purge rate stream 9 & Double \\
\hline CORR of Product separator temperature & Double \\
\hline CORR of Product separator level & Double \\
\hline CORR of Product separator pressure & Double \\
\hline CORR of Product separator underflow stream 10 & Double \\
\hline CORR of stripper level & Double \\
\hline CORR of stripper pressure & Double \\
\hline CORR of Stripper underflow stream 11 & Double \\
\hline CORR of stripper temperature & Double \\
\hline CORR of strippx steam dew & Double \\
\hline CORR of Compressor work & Double \\
\hline CORR of Reactor cooling water outlet temperature & Double \\
\hline CORR of separator cooling water outlet temperature & Double \\
\hline CORR of Reactor feed A & Double \\
\hline CORR of Reactor feed B & Double \\
\hline CORR of Reactor feed C & Double \\
\hline CORR of Reactor feed D & Double \\
\hline CORR of Reactor feed $\mathrm{E}$ & Double \\
\hline CORR of Reactor feed $\mathrm{F}$ & Double \\
\hline CORR of Purge gas $A$ & Double \\
\hline CORR of Purge gas $B$ & Double \\
\hline CORR of Purge gas $C$ & Double \\
\hline CORR of Purge gas $D$ & Double \\
\hline CORR of Purge gas $E$ & Double \\
\hline CORR of Purge gas $F$ & Double \\
\hline CORR of Purge gas $\mathrm{G}$ & Double \\
\hline
\end{tabular}




\begin{tabular}{|l|l|}
\hline Name & Type \\
\hline CORR of Purge gas H & Double \\
\hline CORR of Product D & Double \\
\hline CORR of Product E & Double \\
\hline CORR of Product F & Double \\
\hline CORR of Product G & Double \\
\hline CORR of Product H & Double \\
\hline Corr_to_OpCost & Double \\
\hline MaxDev_from_OpCost & Double \\
\hline Corr_to_PctG & Double \\
\hline MaxDev_from_PctG & Double \\
\hline Var_from_PctG & Double \\
\hline
\end{tabular}

Figure 19- Figure 23 illustrate some computed metrics for reactor pressure, product quality, and cost. Maximum deviation is computed for each disturbance vector and P-R pair, and each is stored separately in a metrics database. By investigating the graphs in Figure 19, it is clear that the reactor pressure deviates significantly from the baseline case as $\mathrm{P}$ exceeds 0.1 and $\mathrm{R}$ remains below 0.18 . The metrics shown in the charts are for all disturbances vectors combined. Although the metrics shown in the figure are computed for all disturbance scenarios, a benefit of storing the metrics in a database is that the metrics can be refined by targeting a specific disturbance vector or set of vectors by modifying the underlying query.

Examination of Figure 20 shows that the $1 \sigma$ deviation of the reactor pressure climbs to approximately $25 \mathrm{kPa}$ when all disturbances vectors are considered, which may be significant to a plant operator who desires to operate the reactor close to the shutdown pressure threshold of 3,000 kPa. Only the plant operator can determine how significant such a deviation is to plant operations.

Another key performance metric is product quality. Product quality is measured as a molar percentage of the overall production output. The product quality metric is computed here as a deviation to the baseline case. Figure 21 shows the product quality metrics aggregated for all disturbance types. As a sanity check, the baseline case is shown in the bottom right graph for $P=0, R=1$ to have zero molar percent deviation from the baseline case. All other charts show a deviation in chemical composition of the process output between $0.6 \%$ and $0.8 \%$. The significance of these deviations is determined by the requirements of the downstream process which was not described by Downs and Vogel. [7]

Operating cost is a significant metric to process operators and is the final metric presented here. The operating cost metric is presented as a correlation between the baseline case and the test cases. Correlation, calculated as a dot product between two vectors, provides a perspective on how well one signal tracks another assuming that the signals follow a linear regression. A correlation value of 1 indicates the baseline case and the test case track identically. A correlation of zero indicates that the baseline and test cases do not seem to be linked. A negative correlation indicates that the test cases diverge from the baseline case. Shown in Figure 22 are correlations between the baseline operating cost and the operating cost of each test case across all disturbance types for progressively increasing $P$ and $R$ values. For values of $R>0.1$, the data indicates that the operating cost remains relatively unchanged even for values of $P$ approaching unity. For values of $\mathrm{R}>0.5$, it is clear that the test cases can be considered equivalent to the 
baseline case. This indicates that a high recovery probability is essential to maintaining expected operating costs.

While correlation of operating cost between the baseline case and the test cases is an interesting metric, an operator would still want to understand the cost deviation from baseline. Cost deviation from baseline iss shown in Figure 23. Cost Deviation is calculated as the baseline cost minus the test case cost. While one may expect operating costs to rise if the network is impacts, the opposite is actually true for TEsim. Operating costs are primarily a function of the rate of consumption of raw materials. Raw materials are lost in the purge gas, the product stream, and by two side reactions. A reduction in cost

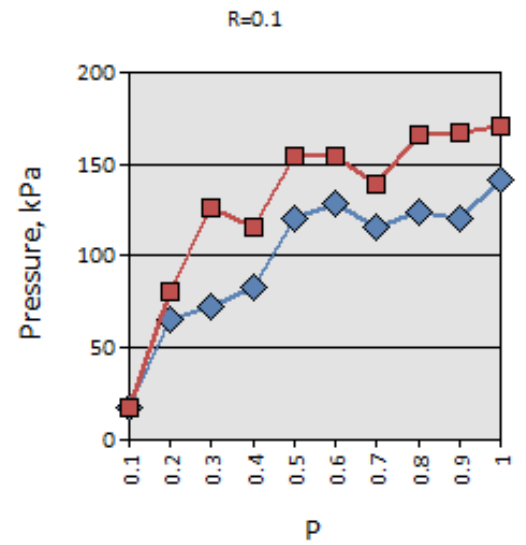

0.18

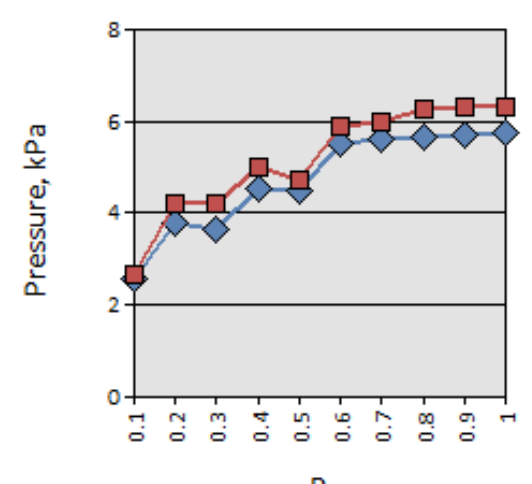

0.14

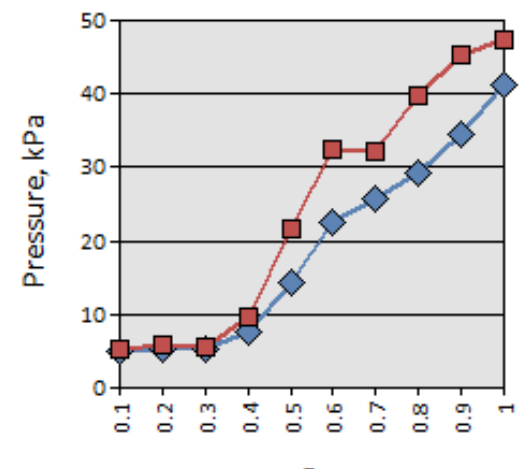

$\mathrm{P}$

0.2

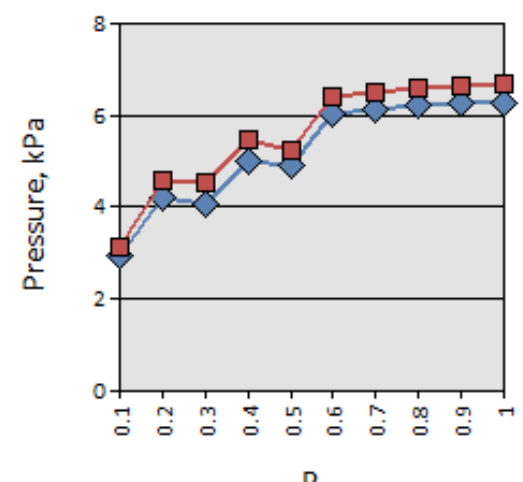

0.16

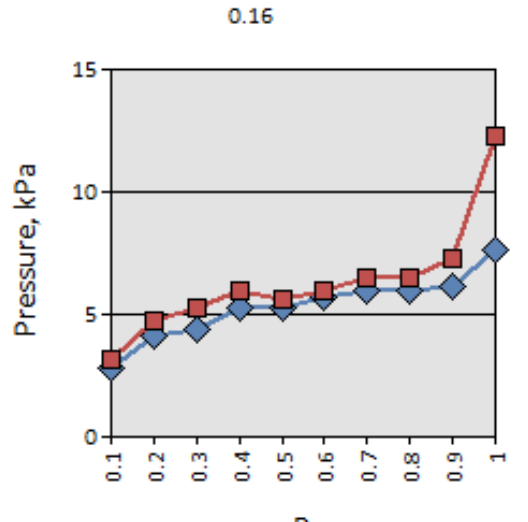

$\mathrm{P}$

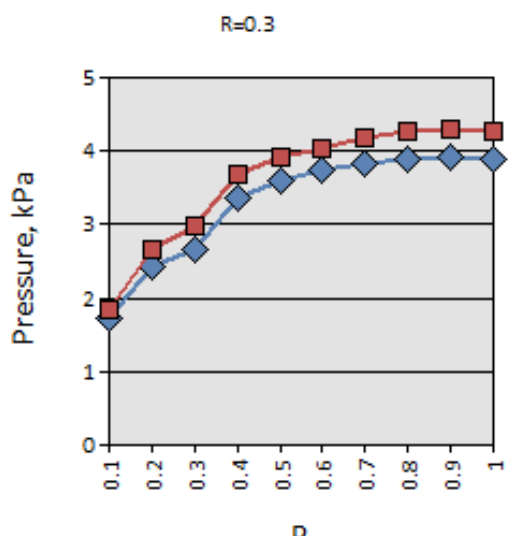

could indicate an undesirable affect to product quality that may affect downstream processes and
ultimately increase costs.

could indicate an undesirable affect to product quality that may affect downstream processes and
ultimately increase costs.

\section{Deviation Metrics for Reactor Pressure}

(n)

(1)




\section{Deviation Metrics for Reactor Pressure}
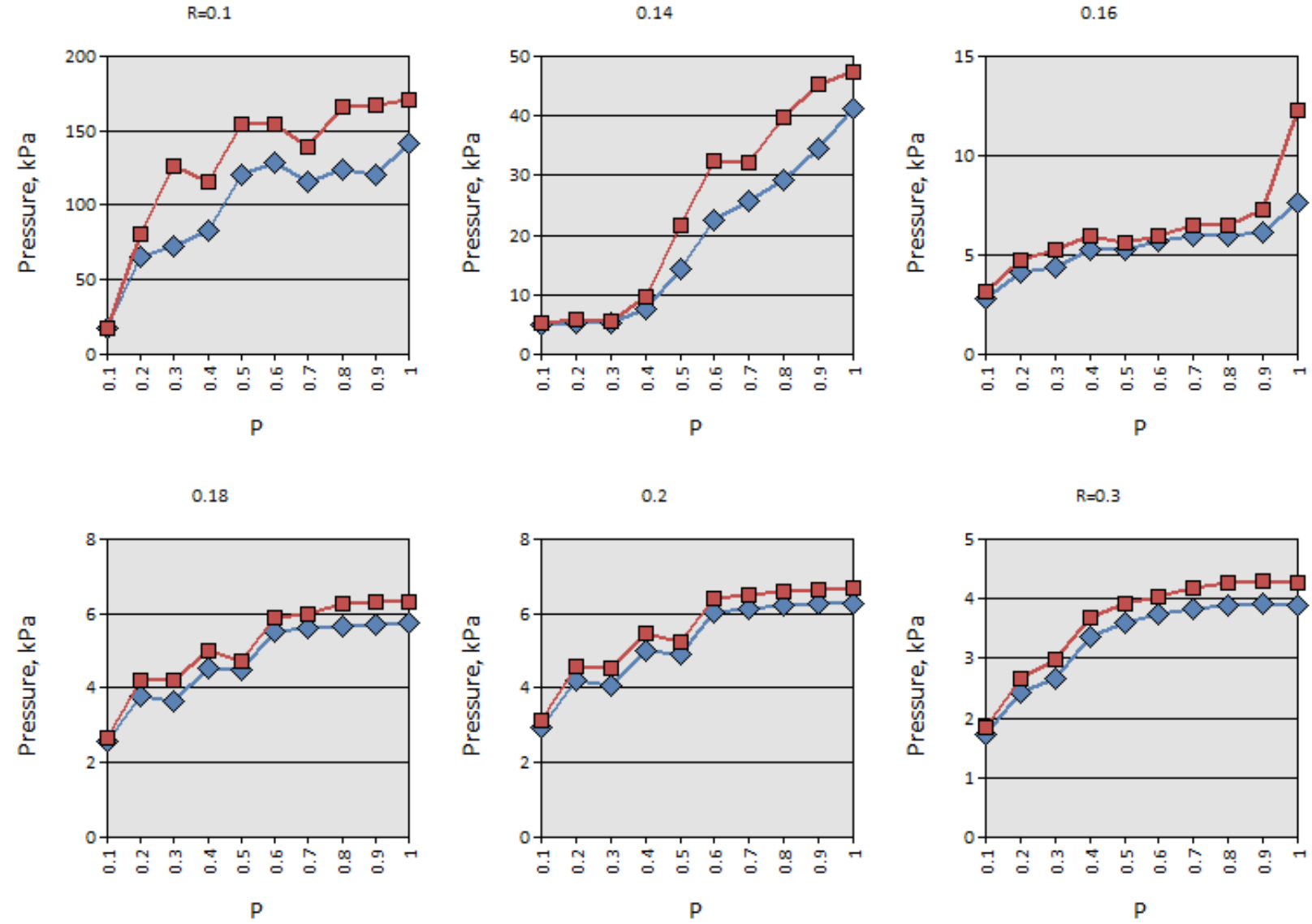

$\neg \checkmark$ Average of MAXDEV from Reactor pressure $\square-$ Max of MAXDEV from Reactor pressure 


\section{Standard Deviation of Maximum Reactor Pressure}

$R=0.1$

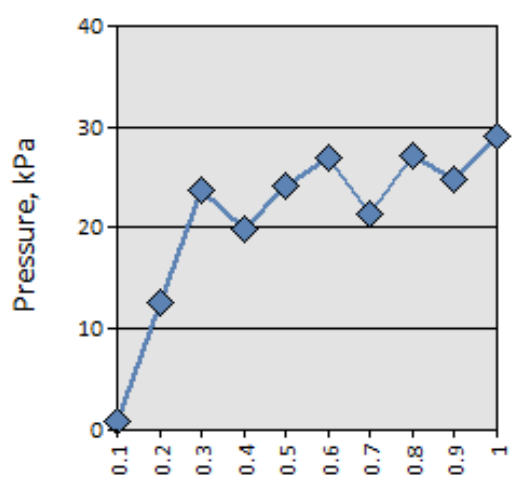

$\mathrm{P}$

0.18

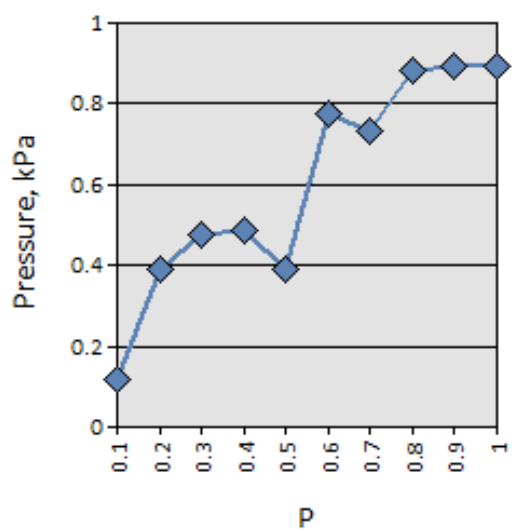

0.14

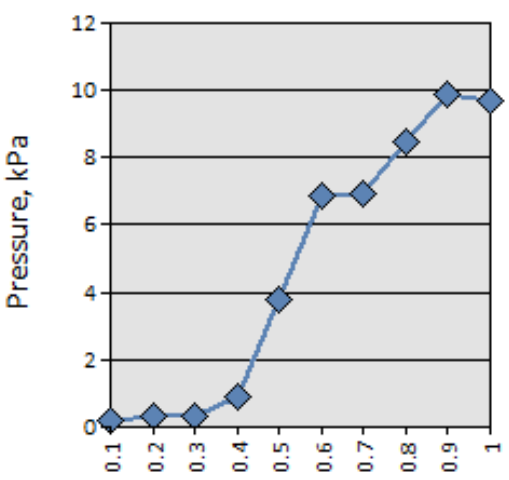

P

0.2

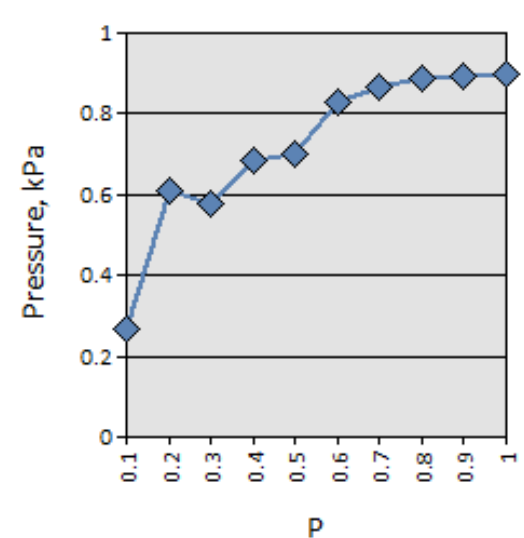

0.16

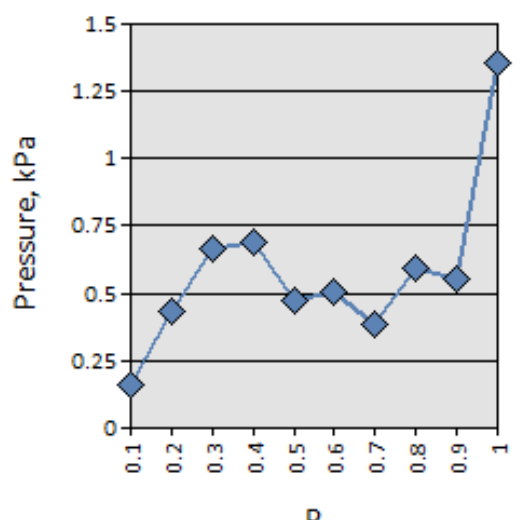

$R=0.3$

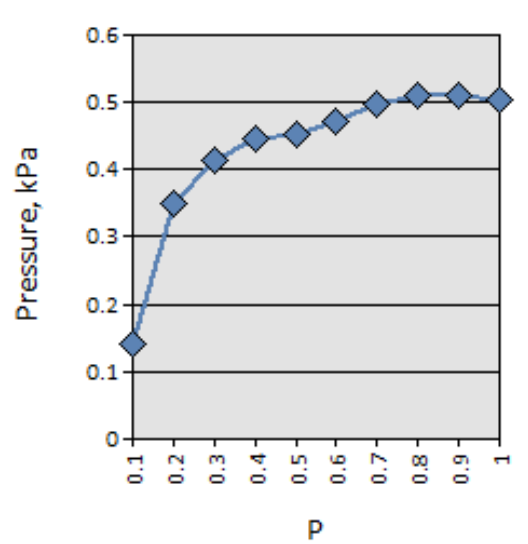

Figure 20. Standard Deviation of the Maximum Reactor Pressure Deviation for All Disturbances 

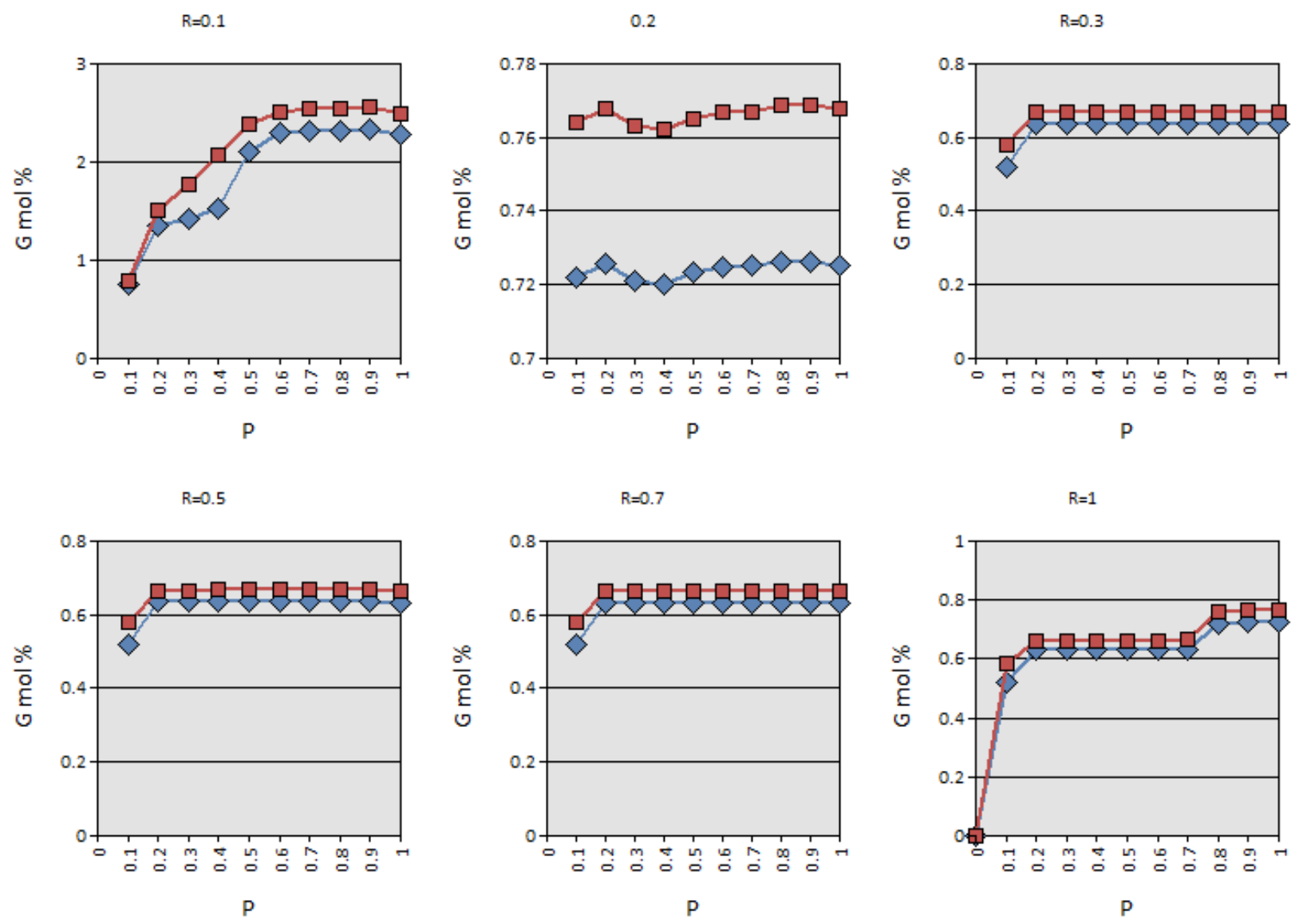

$\checkmark$ Average of MaxDev_from_PctG - - Max of MaxDev_from_PctG

Figure 21. Product Quality Metrics for All Disturbances 
$R=0.1$

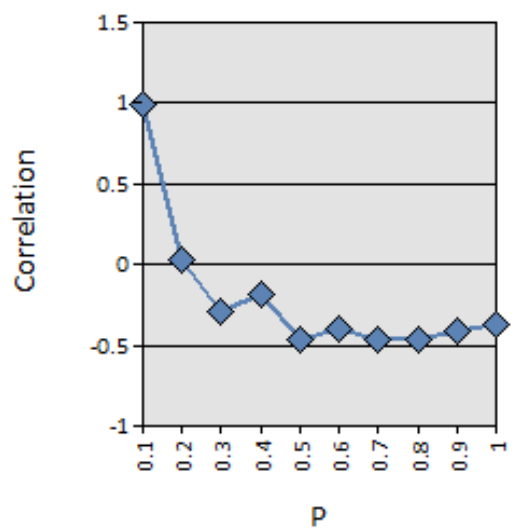

0.16

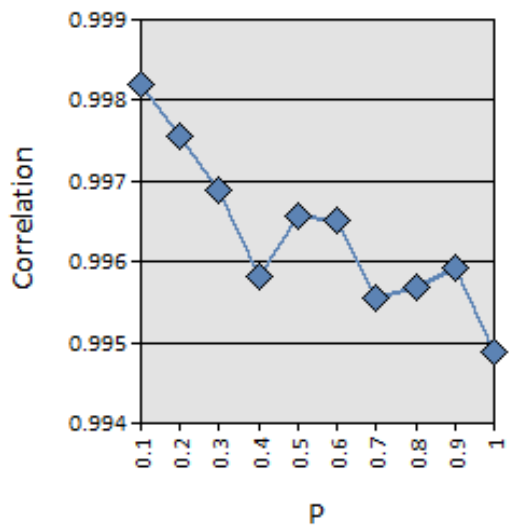

0.12

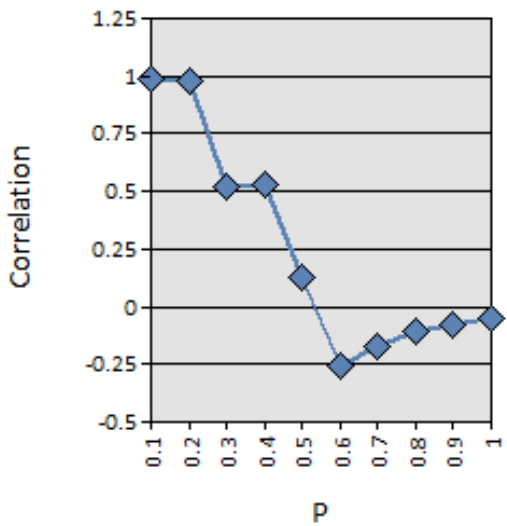

0.18

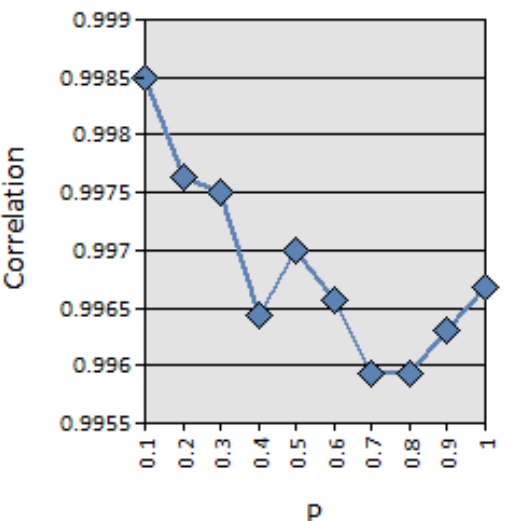

0.14

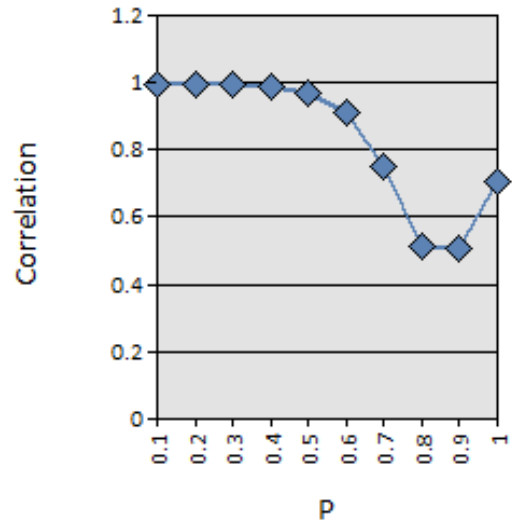

0.2

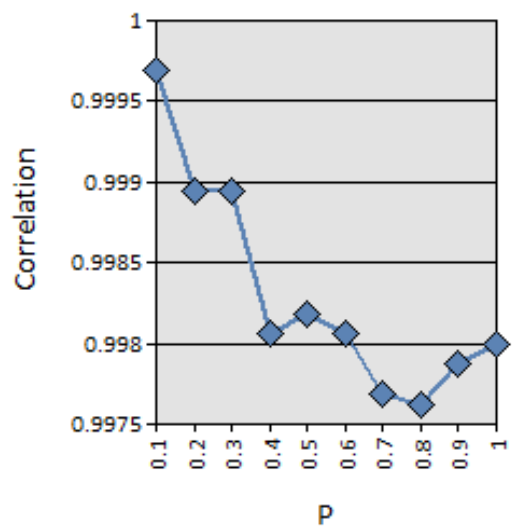

Figure 22. Operating Cost Metric for All Disturbances 
$R=0.1$

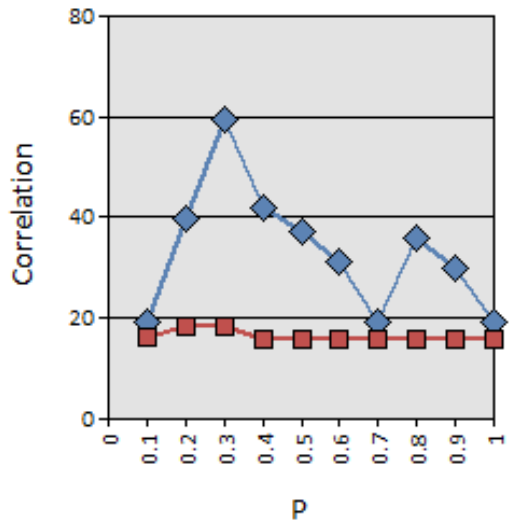

0.18

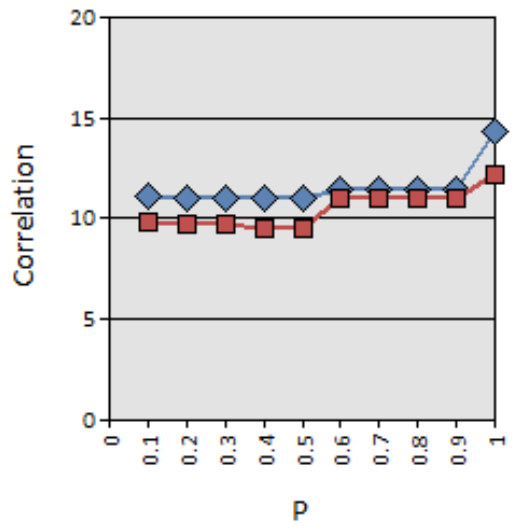

0.12

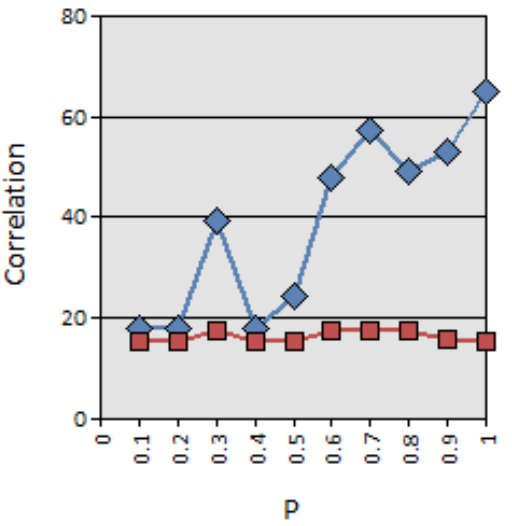

0.2

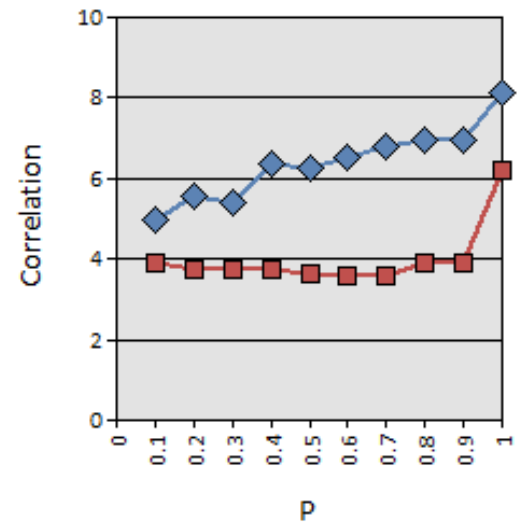

0.14

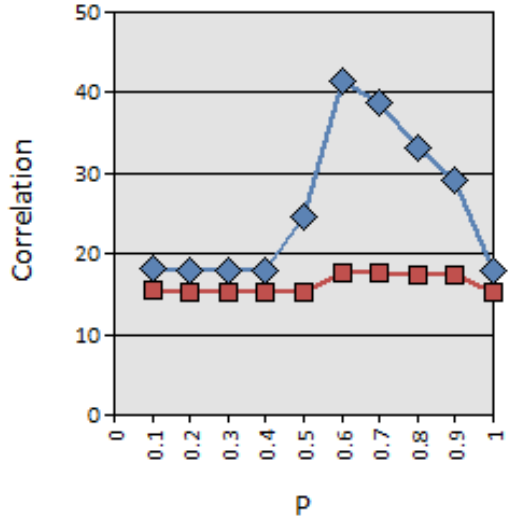

1

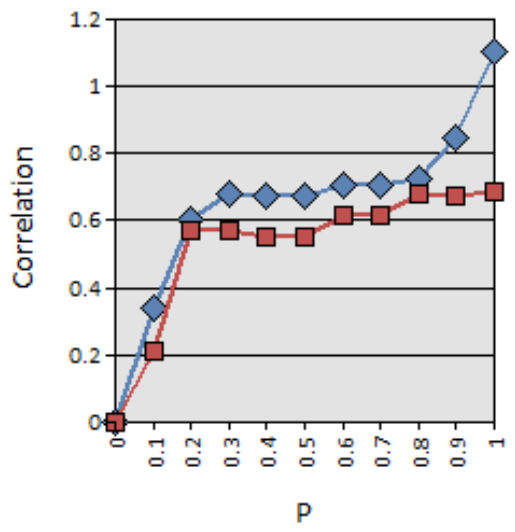

Figure 23. Deviation of Operating Cost from Baseline 


\subsubsection{Conclusions and Recommendations}

The TE model provides a large number of variables by which to measure performance. By applying various disturbances and channel model probabilities, over 2,000 different scenarios were executed. Time series data sets for each of the 41 measured variables and several other indicators for each scenario were generated. The raw output data for each disturbance vector and channel model was stored in a MATLAB formatted data file (i.e., a ".mat" file). Scripts were developed to produce statistical metrics for the data and collect the metrics in both a Microsoft ${ }^{\circledR}$ Excel spreadsheet and a Microsoft ${ }^{\circledR}$ Access database. All code and metrics were stored for public use in the TEsim GitHub repository.

The Tennessee Eastman process can be said to exhibit slow dynamics properties, and much of the model as it is implemented can be considered of zero order. However, this does not mean that the "realworld" equivalent would have no dynamic properties. A real world process would exhibit physical oscillations (temperature, pressure, and composition) due to physical and chemical processes. Considering the widespread acceptance of the TE process model by industry and academia, the TE model as it is implemented represents the real-world process accurately enough to measure the effects of a generic network security control on the process.

The TEsim results indicate that a simple Gilbert channel between plant and controller does indeed impact process performance when the probability of transition from the good state to the bad state appreciably exceeds zero and the recovery probability, R, remains very low. It is incumbent upon the system integrator to select a network configuration and device capabilities that can accommodate the number of sensors, the number of actuators, and the security policies to be enforced. Enforcement of security may include deep packet inspection, authentication, and encryption all of which will introduce a processing load on the device. It can therefore be recommended that the devices be chosen such that the communications channel between plant and controller is operated in a P-R region appropriate for the application.

Deployment of perimeter security devices to existing industrial applications requires the operator to first ascertain the need for security. This should be performed in accordance with NIST SP 800-82 or an associated standard. If a security posture is required based on the outcome of the risk assessment, the operator must ascertain the impact of security controls on the performance of the industrial process being instrumented assuming that the deployed protocols are compatible with available security devices. Many legacy operations may simply require security controls implemented at the boundary between the ICS and the corporate network or public internet. Other ICSs may require security controls between sensor/actuator space and the controller (i.e., the PLC) as was implemented in TEsim model. Once a set of security controls is selected for the ICS, a network architecture must be developed (if the existing architecture can be modified) and security devices must be selected. The security devices should be selected with sufficient processing and memory capabilities to support the intended protocols, traffic, scan rate (if applicable), algorithms, and set of security rules (if applicable). If it is unclear how performance will be affected using the data provided by the device manufacturer, device characterization and simulation is recommended. ${ }^{10}$ The GE channel method that was applied to the TE process is one proposed method to measure performance in simulation. Using the TEsim method would require the system integrator to characterize each security device with the protocols and rules intended. This

\footnotetext{
${ }^{10}$ A standard test methodology does not yet exist for security device manufacturers and ICS system integrators to measure the performance of a security device as it relates to ICS performance impact assessment.
} 
approach implies the need for a test method to characterize ICS security devices, yet no NISTrecommended method yet exists for this purpose.

Using TEsim, it was demonstrated that for the Tennessee Eastman processes in which sensor and actuators were scanned at a rate of $1 \mathrm{~Hz}$ that the channel could tolerate some congestion through the security device if the recovery probability was high enough. As stated, the TE process is slow acting and can tolerate some interruption between plant and controller. Other processes may not be as tolerant to interruption. Examples of such processes may include a robotic assembly process, high-speed conveyor operations, and safety applications. Only the plant operator has the knowledge to ascertain the impact of channel congestion on plant performance.

As previously stated, the number of sensors and actuators will impact the load on any aggregating network device including switches, router, and firewalls. Logically grouping networked devices that communicate through a security device may serve as a method to alleviate congestion in a single firewall. While this approach will increase the number of devices to be managed (e.g., rule deployment and key distribution) it could facilitate the enforcement of a stronger security policy on the network or make the network safer to operate.

From a security perspective, it is recommended that the network topology, security devices, and policies be selected appropriate to the estimated security risk in accordance with NIST SP 800-82. It is further recommended that the devices and network topology selected be deployed with sufficient processing headroom should a network-based attack occur. Few industrial network attacks will add significant load to a network channel. Denial-of-service (DoS) attacks are obvious attack types that add significant load to the network as this is the strategy of the DoS attack. Other more stealthy attacks could overload the network channel by putting the security device into a state in which specific rules are executed excessively. Security rules should be designed in such a way that the device cannot be driven into an overload state. Depending on the estimated risk, an accurate software-based simulation that includes realistic estimates of the heuristics load of filtering devices may be used to ascertain the impact of security controls on the performance of the industrial process. Estimating heuristic load is entirely different topic of interest for the ICS cybersecurity community. 